\title{
Low-Frequency Variability in the Midlatitude Baroclinic Atmosphere Induced by an Oceanic Thermal Front
}

\author{
Yizhak Feliks, ${ }^{*}$ Michael Ghil, ${ }^{+}$and Eric Simonnet \\ Department of Atmospheric and Oceanic Sciences, and Institute of Geophysics and Planetary Physics, University of California, \\ Los Angeles, Los Angeles, California
}

(Manuscript received 14 September 2005, in final form 5 March 2006)

\begin{abstract}
This study examines the flow induced by an east-west-oriented oceanic thermal front in a highly idealized baroclinic model. Previous work showed that thermal fronts could produce energetic midlatitude jets in an equivalent-barotropic atmosphere and that barotropic instabilities of this jet had dominant periods of 25-30 and $65-75$ days.

The present study extends this work to a two-mode baroclinic free atmosphere. The baroclinic jet produced in this case is subject to both barotropic and baroclinic instabilities. A barotropic symmetric instability propagates westward with periods of roughly 30 days and is similar to those found in the equivalent-barotropic model. A baroclinic instability results in standing-dipole anomalies and oscillates with a period of 6-8 months. A mixed barotropic-baroclinic instability results in anomalies that propagate northward, perpendicular to the jet, with a period of 2-3 months. The later anomalies are reminiscent of the 70-day oscillation found over the North Atlantic in observed fields.

The atmospheric flow has two distinct states: the flow in the high-energy state exhibits two large gyres and a strong eastward jet; its antisymmetric component is dominant. The low-energy flow is characterized by small gyres and a weak jet.

The model's dynamics depends on the layer-depth ratio. When the model is nearly equivalent-barotropic, symmetric oscillatory modes dominate. As the two layers become nearly equal, antisymmetric oscillatory modes become significant and the mean energy of the flow increases.

When the oceanic thermal front's strength $T^{*}$ is weak $\left(T^{*} \leq 1.5^{\circ} \mathrm{C}\right)$, the flow is steady. For intermediate values of the strength $\left(1.5^{\circ} \mathrm{C}<T^{*}<3^{\circ} \mathrm{C}\right)$, several oscillatory instabilities set in. As the frontal strength increases further $\left(T^{*} \geq 3^{\circ} \mathrm{C}\right)$, the flow becomes more turbulent. These results all depend on the atmospheric model's horizontal resolution being sufficiently high.
\end{abstract}

\section{Introduction}

The strongest thermal contrasts at the ocean's surface occur across major oceanic currents and upwelling zones. Midoceanic thermal fronts, such as the Gulf Stream and Kuroshio Extension, are permanent features of the midlatitude ocean circulation (Stommel

\footnotetext{
* Additional affiliation: Mathematics Department, Israel Institute of Biological Research, Nes-Ziona, Israel.

+ Additional affiliation: Département Terre-AtmosphéreOcéan, Ecole Normale Supérieure, and Laboratoire de Météorologie Dynamique du CNRS, IPSL, Paris, France.
}

Corresponding author address: Eric Simonnet, Institut NonLinéaire de Nice, CNRS, 1361, route des Lucioles, 06560 Valbonne, France.

E-mail: eric.simonnet@inln.cnrs.fr
1965; Stommel and Yoshida 1972). These fronts change in position and strength on the time scale of weeks to years. The sea surface temperature (SST) gradient across these fronts is of about $T^{*}=4^{\circ}-10^{\circ} \mathrm{C}(100 \mathrm{~km})^{-1}$ (Sweet et al. 1981). Most of the data on these fronts are derived from a limited number of in situ hydrographic sections and a much larger number of remotely taken, satellite infrared images (Lee and Cornillon 1996).

The response of the atmospheric marine boundary layer (AMBL) to oceanic fronts has been studied in observations, as well as by analytical and numerical models; see Feliks et al. (2004, hereafter F04) for a review. Most previous studies concentrated on steadystate AMBL solutions (Hsu 1987) or on the AMBL's short-time evolution (of the order of 12-24 h; e.g., Warner et al. 1990).

F04 concentrated instead on the low-frequency variability (LFV) in the atmosphere induced by a steady

DOI: $10.1175 / \mathrm{JAS} 3780.1$

(C) 2007 American Meteorological Society 
SST front. They studied the flow induced by an eastwest-oriented SST front of finite zonal extent (see Fig. 1) in a highly idealized model of the midlatitude atmosphere. A very simple, linear model of the AMBL was coupled to a quasigeostrophic (QG), equivalentbarotropic nonlinear model of the free atmosphere.

The extreme simplicity of the model allowed us to focus on a previously neglected, but potentially important effect of such an SST front, namely the role of potential vorticity (PV) injection into the free atmosphere. In traditional atmospheric models of oceanatmosphere interaction, no net PV source due to lowerboundary heating is taken into account (see Kushnir et al. 2002). In the F04 model, the vertical velocity at the top of the AMBL drives the flow in the free atmosphere and produces an eastward jet, parallel to the oceanic front's isotherms. A large gyre develops on either side of this jet, cyclonic poleward and anticyclonic equatorward of it.

The behavior of this jet-and-gyres flow field depends on the thermal gradient $T^{*}$ across the front, for a given length of the front. When this length is $600 \mathrm{~km}$, as in Fig. 1, the flow is steady, with a velocity field that is symmetric about the axis of the oceanic front, as long as $T^{*} \leq 4^{\circ} \mathrm{C}$. We refer to such a pattern as antisymmetric, because the barotropic streamfunction of the free atmosphere has this property. For $4^{\circ} \mathrm{C}<T^{*}<5^{\circ} \mathrm{C}$, the flow is periodic in time, with a period of roughly 30 days, and it is asymmetric in space. The Hopf bifurcation at $T^{*} \cong 4^{\circ} \mathrm{C}$ that leads from steady to periodic flows is caused by a shear instability with a characteristic wavelength of about $500 \mathrm{~km}$ and a symmetric streamfunction field. The superposition of this symmetric pattern onto the antisymmetric basic flow results in the overall asymmetry of the full oscillatory solution.

At $T^{*} \simeq 5^{\circ} \mathrm{C}$ the solution becomes chaotic and its dominant period is still close to a month. This period increases suddenly to over two months at $T^{*} \simeq 6^{\circ} \mathrm{C}$. The two flow regimes, below and above $T^{*} \simeq 6^{\circ} \mathrm{C}$, possess distinct characteristics, besides the dominant period: (i) The former one is a low-energy regime, in which the SST front, and hence the atmospheric jet, are relatively weak; in this regime, small meanders develop along the jet axis. (ii) The latter is a high-energy regime in which the SST front and the jet are stronger; in it, large meanders and eddies develop along the jet.

In the F04 model, only barotropic instability mechanisms are present, while baroclinic instability played a major role in earlier studies of the midlatitude atmosphere's response to SST anomalies (Kushnir et al. 2002). In the present study we use a baroclinic model of the free atmosphere in order to examine the role of baroclinic instabilities in the jet-and-gyres dynamics.

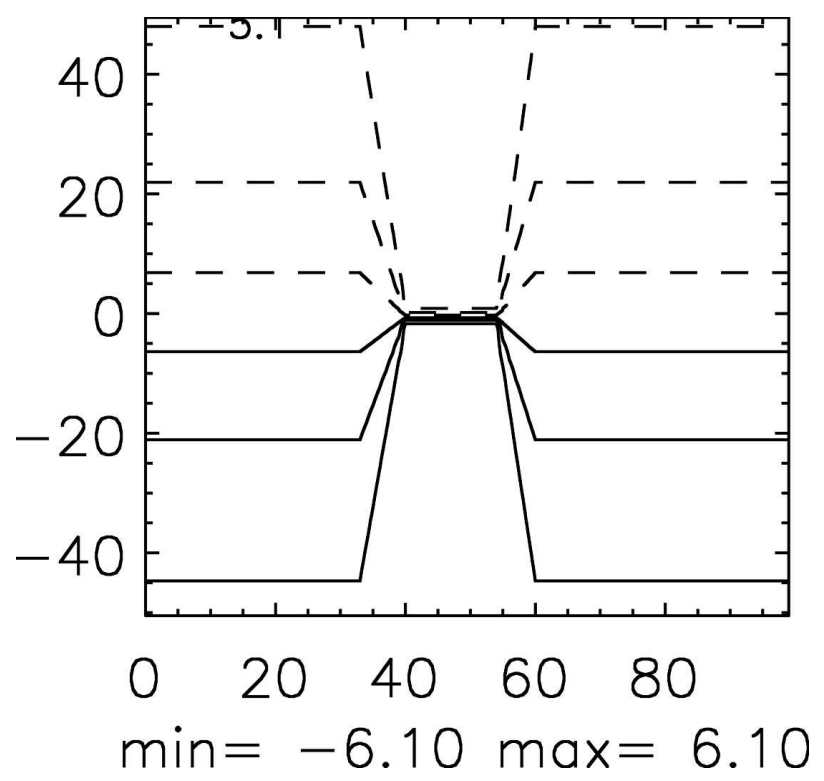

FIG. 1. Prescribed SST pattern for an oceanic front of length 600 $\mathrm{km}$ with strength $T^{*}=6.1^{\circ} \mathrm{C}$ and frontal-width parameter $d=50$ $\mathrm{km}$; see Eq. (15). Contour interval (CI) is $2^{\circ} \mathrm{C}$, starting at $\pm 6^{\circ} \mathrm{C}$; positive contours are solid; negative and zero contours dashed. Axes in nondimensional units of $\Delta x$ counts, where $\Delta x=\Delta y=50$ $\mathrm{km} / L, L$ being the length scale; see Eq. (1).

More precisely, the purpose of this work is to provide the answer to the following three questions:

1) Does the jet induced by the SST front in a barotropic atmosphere survive in a baroclinic atmosphere, where baroclinic instabilities are dominant?

2) What is the time scale and structure of the baroclinic instabilities?

3) Are there any mixed barotropic-baroclinic instabilities, and, if so, what do they look like?

A key motivation of the theoretical study in F04 and here is to elucidate the origin of intraseasonal atmospheric oscillations observed over the North Atlantic Ocean. In particular, the 70-day oscillation found by Plaut and Vautard (1994) in geopotential heights at 700 $\mathrm{hPa}$ from the National Meteorological Center (NMC) is characterized by fluctuations in both amplitude and position of the Atlantic jet, with a poleward-propagating anomaly pattern. This oscillation cannot be related to topographic instabilities, as is the case for other midlatitude intraseasonal oscillations (Simmons et al. 1983; Ghil and Childress 1987; Jin and Ghil 1990), nor to the extratropical effects of the Madden and Julian (1971) oscillation. Our study does provide two plausible mechanisms for this oscillation, both related to instabilities induced by the strong SST gradients over the Gulf Stream. 
A further motivation of our work is to provide a clearer understanding of the links between the Gulf Stream position and strength, on the one hand, and the North Atlantic Oscillation (NAO; Hurrell 1995; Hurrell et al. 2003) and the frequency distribution of weather regimes (Kimoto and Ghil 1993a,b; Plaut and Vautard 1994; Robertson et al. 2000) over the North AtlanticEuropean sector, on the other. These links, however, will have to be pursued further elsewhere, given the highly idealized character of the models used by F04 and here.

In section 2, the baroclinic QG model of the free atmosphere is described. In section 3, we study the model's instabilities, and the LFV and ultralow LFV to which they give rise. In section 4 , we summarize and discuss these results.

\section{The atmospheric model}

We consider an atmospheric model composed of a steady, analytical AMBL and a time-dependent, QG baroclinic model for the free atmosphere above it. The AMBL was described in details in F04. We present here the baroclinic model for the free atmosphere.

This QG model has two modes in the vertical and the model equations are nondimensionalized by the following characteristic scales:

$$
X, Y \propto L ; Z \propto H ; T_{i} \propto L / V ; \psi \propto V L ; T \propto T^{*} .
$$

Here, $L$ is the horizontal length scale across the front, $H$ is the vertical scale of the free atmosphere, $T_{i}$ is the advective time scale, $V$ scales the wind speed in the cross-front direction, and the (nondimensional) temperature $T$ scales like the oceanic frontal strength $T^{*}$. In our experiments, $T^{*}$ will be varied; as seen later, this is equivalent to varying the SST gradient of the thermal front. The appropriate scales are listed in Table 1, along with the main physical parameters.

The nondimensional PV equation with this scaling is

$$
\frac{\partial q}{\partial t}+\beta \frac{\partial \psi}{\partial x}+J(\psi, q)=r_{H} \nabla^{4} \psi,
$$

where $w_{a}$ is the vertical velocity in the free atmosphere (Pedlosky 1987; Holton 1992).

The relative $\mathrm{PV}$ is given by

$$
q \equiv \nabla^{2} \psi+\frac{\partial}{\partial z}\left(\frac{1}{S} \frac{\partial \psi}{\partial z}\right)
$$

and the nondimensional parameters $S, \beta$, and $r_{H}$ by

$$
S=\frac{N^{2} H^{2}}{f^{2} L^{2}}, \beta=\beta_{0} \frac{L^{2}}{V}, r_{H}=\frac{E_{H}}{2 \epsilon}=\frac{K_{H}}{V L} ;
$$

TABLE 1. Characteristic scales and reference values for the atmospheric model.

\begin{tabular}{lc}
\hline \hline$L(\mathrm{~km})$ & 50 \\
$H(\mathrm{~km})$ & 10 \\
$H_{a}(\mathrm{~km})$ & 10 \\
$H_{E}(\mathrm{~km})$ & 0.8 \\
$T_{i}(\mathrm{day})$ & 0.58 \\
$V\left(\mathrm{~m} \mathrm{~s}^{-1}\right)$ & 1 \\
$\beta_{0}\left(\mathrm{~m}^{-1} \mathrm{~s}^{-1}\right)$ & $1.8 \times 10^{-11}$ \\
$k_{0}\left(\mathrm{~m}^{2} \mathrm{~s}^{-1}\right)$ & 3.3 \\
$K_{H}\left(\mathrm{~m}^{2} \mathrm{~s}^{-1}\right)$ & $10^{2}$ \\
$f\left(\mathrm{~s}^{-1}\right)$ & $10^{-4}$ \\
$g\left(\mathrm{~m} \mathrm{~s}^{-2}\right)$ & 9.81 \\
$\theta_{0}(\mathrm{~K})$ & 300 \\
$\left.\rho_{0}(\mathrm{~g} \mathrm{~cm})^{-3}\right)$ & $10^{-3}$ \\
$L / \lambda_{1}(\mathrm{~km})$ & 575 \\
\hline
\end{tabular}

$S$ is the mean Burger number, $\beta_{0}$ is the meridional gradient of $f, N=N(z)$ is the Brunt-Väisälä frequency, $K_{H}$ is the dimensional horizontal diffusion coefficient, $\varepsilon=$ $V / f L$ is the Rossby number, based on the cross-frontal speed $V$, and $E_{H}=2 K_{H} / f L^{2}$ is the horizontal Ekman number. We shall also use the Froude number $F_{r}=$ $V\left(g H_{E} T^{*} / \theta_{0}\right)^{-1 / 2}$ of the AMBL, where $\theta_{0}$ is the (dimensional) reference value of the virtual potential temperature. The height of the AMBL is $H_{E}=\pi\left(2 k_{0} / f\right)^{1 / 2}$, where $k_{0}$ is the turbulent eddy coefficient in the vertical direction (see F04 for further details).

Along the meridional boundaries, we impose freeslip $\psi=q=0$, while in the zonal direction we use the open boundary condition of Charney et al. (1950); that is, the vorticity is specified in the inflow points and the streamfunction is specified everywhere. Following F04, the lower boundary condition for the free atmosphere at $z=0$ is

$$
\begin{aligned}
\frac{H}{H_{a}} \frac{d}{d t}\left[\frac{\partial}{\partial z}\left(\frac{1}{S} \frac{\partial \psi}{\partial z}\right)\right] & =\frac{H}{H_{a}} w_{a}(x, y, z=0, t) \\
& =\gamma \nabla^{2} \psi-\alpha \nabla^{2} T,
\end{aligned}
$$

where $H_{a}$ is the height of the free atmosphere, nondimensionalized in Eq. (5) by $H$. The vertical velocity $w_{a}$ has, therewith, two components: one is due to the geostrophic flow above the AMBL, called in F04 the mechanical component; the other is due to the wind induced in the AMBL by the oceanic thermal front and called the thermal component.

The nondimensional constant

$$
\alpha=\frac{1}{2 \pi}\left(1-\frac{1}{2 \pi}\right) \frac{g H_{E}^{2}}{H_{a} V^{2}} \frac{T^{*}}{\theta_{0}}=\frac{1}{2 \pi}\left(1-\frac{1}{2 \pi}\right) \frac{H_{E}}{H_{a}} \frac{1}{F_{r}^{2}}
$$


determines the strength of the thermal component of the vertical velocity component at the top of the AMBL. This component is proportional to the inverse square Froude number of the AMBL, as well as to $H_{E}^{2}$ (or equivalently $k_{0}$ ) and $T^{*}$. The turbulent eddy coefficient $k_{0}$ will be kept constant, whereas $T^{*}$ will be varied systematically.

The nondimensional constant

$$
\gamma=\frac{1}{2 \pi} \frac{H_{E}}{H_{a}} \frac{f L}{V}=\frac{1}{2 \pi \epsilon} \frac{H_{E}}{H_{a}}
$$

determines the strength of the mechanical component of the vertical velocity at the top of the AMBL. It is proportional to the inverse of the frontal Rossby number. We see that $\alpha \propto 1 / F_{r}^{2}, \gamma \propto 1 / \epsilon$, and both are proportional to $H_{E} / H_{a}$.

An efficient discretization in the vertical of our model's free atmosphere is based on the orthonormal eigenfunctions $\phi_{k}(z)$ of the eigenvalue problem

$$
\frac{\partial}{\partial z}\left(\frac{1}{S} \frac{\partial \phi_{k}}{\partial z}\right)=-\lambda_{k}^{2} \phi_{k},
$$

following Flierl (1978) and Feliks and Ghil (1996). The appropriate boundary conditions are $\partial \phi_{k} / \partial z=0$ at the lower boundary $z=0$ (the top of the AMBL), as well as at $z=1$, taken to represent the mean height of the tropopause. We designate by $k=0$ the barotropic mode and by $k \geq 1$ the successive baroclinic modes. The external Rossby radius of deformation $L / \lambda_{0}$ is infinite, according to the rigid-lid assumption; the $k$ th internal Rossby radius of deformation is $L / \lambda_{k}$ and we shall retain the first two modes only, that is $k=0,1$. This limited vertical resolution still allows us to capture the main barotropic and baroclinic processes of interest, as well as the interactions between the two modes.

We decompose the streamfunction $\psi$ and the PV $q$ into vertical normal modes, namely

$$
\begin{aligned}
\psi(x, y, z, t) & =\sum_{k} \psi_{k}(x, y, t) \phi_{k}(z) \\
q(x, y, z, t) & =\sum_{k} q_{k}(x, y, t) \phi_{k}(z) .
\end{aligned}
$$

The equation for the modal amplitudes is (Flierl 1978):

$$
\begin{aligned}
& \frac{\partial q_{k}}{\partial t}+\beta \frac{\partial \psi_{k}}{\partial x}+ \sum_{i, j} \xi_{i j k} J\left(\psi_{i}, q_{j}\right)= \\
& r_{H} \nabla^{4} \psi_{k}-\frac{H}{H_{a}} \phi_{k}(0) w_{a}(z=0),
\end{aligned}
$$

where we only retain $k=0,1$ and

$$
q_{k}=\nabla^{2} \psi_{k}-\lambda_{k}^{2} \psi_{k}, \quad \xi_{i j k}=\int \phi_{i} \phi_{j} \phi_{k} d z .
$$

The triple interaction coefficients $\xi_{i j k}$ are all equal to zero or one except $\xi_{111}$; we shall refer to the value of $\xi_{111}$ by $\xi$.

The correspondence between two-mode and twolevel models (Flierl 1978) is given by

$$
H_{1}=\frac{\left(\sqrt{\xi^{2}+4}-\xi\right)^{2}}{4+\left(\sqrt{\xi^{2}+4}-\xi\right)^{2}},
$$

where $H_{1}$ is the nondimensional upper-layer depth and $H_{2}=1-H_{1}$ is the lower-layer one; $\xi=0$ gives $H_{1}=$ $H_{2}=1 / 2$, whereas large negative values of $\xi$ give $H_{2}=$ $1-H_{1} \sim 1 / \xi^{2}$. In the basic experiment $\xi=-1$, which corresponds to the values $H_{1} H=7230 \mathrm{~m}$ and $H_{2} H=$ $2770 \mathrm{~m}$, given $H=10000 \mathrm{~m}$ (see Table 1); values of $\xi \simeq-1$ or $H_{2}: H_{1} \simeq 3 / 7$ are often used in atmospheric models (e.g., Kravtsov et al. 2005). In section 4a, the influence of the relative layer depth on the dynamics will be examined.

The modal energy equation is derived by multiplying (10) by $-\psi_{k}$ (Feliks 1990). After some mathematical manipulations, we obtain

$$
\begin{aligned}
\frac{\partial E_{k}}{\partial t}+\sum_{i} e_{i k}+\nabla \cdot \mathbf{P}_{k} & =-\psi_{k} r_{H} \nabla^{4} \psi_{k}+\psi_{k} \phi_{k}(0) w_{a}(z=0), \\
E_{k} & =\frac{1}{2}\left[\left(\nabla \psi_{k}\right)^{2}+\lambda_{k}^{2} \psi_{k}^{2}\right], \\
e_{i k} & =J\left(\psi_{i}, \psi_{k}\right) \sum_{j} \xi_{i j k} q_{j}, \\
\mathbf{P}_{k} & =\left(-\psi_{k} \frac{\partial^{2} \psi_{k}}{\partial x \partial t}-\frac{\beta}{2} \psi_{k}^{2}+\sum \xi_{i j k} q_{i} \psi_{k} \frac{\partial \psi_{i}}{\partial y},-\psi_{k} \frac{\partial^{2} \psi_{k}}{\partial y \partial t}-\frac{\beta}{2} \psi_{k}^{2}+\sum \xi_{i j k} q_{i} \psi_{k} \frac{\partial \psi_{i}}{\partial x}\right) .
\end{aligned}
$$

The term $e_{i k}$ represents the energy transfer between mode $k$ and mode $i$.
The numerical scheme is the same as in Feliks (1990) and in Feliks and Ghil (1996). Key features include 
fourth-order accurate discretization by finite elements in the horizontal (cf. Haidvogel et al. 1980) and a second-order Adams-Bashforth scheme in time.

\section{Basic numerical results}

\section{a. Spinup experiment}

To motivate this study, we consider a strong thermal front that shares certain characteristics with the Gulf Stream and the Kuroshio Extension. The length of the front is $L_{x}=600 \mathrm{~km} / L$, its strength is $T_{a}=3^{\circ} \mathrm{C}$, and its width is $d=50 \mathrm{~km} / L$. These values give a temperature change of roughly $6^{\circ} \mathrm{C}(200 \mathrm{~km})^{-1}$, for the hyperbolictangent profile of Eq. (14) below. The dimensional layer depths are $H_{1} H=7230 \mathrm{~m}$ and $H_{2} H=2770 \mathrm{~m}$, while the other parameters are listed in Table 1.

The computational domain size is $5000 \mathrm{~km} / L \times 5000$ $\mathrm{km} / L$, with a grid size of $\Delta x=\Delta y=50 \mathrm{~km} / L$, and a time step of $T_{i} \Delta t=0.009259$ day $=800 \mathrm{~s}$. The domain is slightly larger than in F04, where the main computations were carried out in a domain that was $4000 \mathrm{~km}$ long in the zonal direction versus $3500 \mathrm{~km}$ wide in the meridional direction. The domain size used here has, a fortiori, a sufficiently large zonal extent to capture the atmospheric response to the given SST field; see additional computations for a greater zonal extent in F04 (their Fig. 14 and section 5b).

F04 showed that both the intensity and variability of the atmospheric flow induced by the oceanic front depended strongly on the horizontal resolution of the calculations. In particular, a sufficiently fine grid is necessary to describe accurately the Laplacian $\nabla^{2} T$ of the SST field and its effect on the atmospheric flow above. To capture, with satisfactory accuracy, the influence of the oceanic front on the evolution of our atmospheric model at the resolution of $50 \mathrm{~km} / L, F 04$ showed, by comparison with an analytical solution, how to adjust the strength $T_{a}$ of the oceanic front, and replace it by $T^{*}=$ $A T_{a}$, where $A=2.06$. Equation (6) is modified accordingly; see Eq. (23) and its discussion in F04 for more details.

The SST front used in this study has the same spatial pattern as in F04:

$$
T(x, y)=-T^{*} \tanh [y / a(x)]
$$

here

$$
a(x)=\left\{\begin{array}{lc}
D, & x \leq x_{0}, \\
d+\frac{x-x_{1}}{x_{0}-x_{1}}(D-d), & x_{0} \leq x \leq x_{1}, \\
d, & x_{1} \leq x \leq x_{2}, \\
d+\frac{x-x_{2}}{x_{3}-x_{2}}(D-d), & x_{2} \leq x \leq x_{3}, \\
D, & x_{3} \leq x,
\end{array}\right.
$$

while $d=50 \mathrm{~km} / L=1, D=2000 \mathrm{~km} / L=40 d=40, x_{0}$ $=1700 \mathrm{~km} / L=34, x_{1}=2000 \mathrm{~km} / L=41, x_{2}=2600$ $\mathrm{km} / L=53$, and $x_{3}=3000 \mathrm{~km} / L=60$. The oceanic thermal front has a length of $600 \mathrm{~km}$ and its SST field is shown in Fig. 1, for a strength of $T^{*}=6.1^{\circ} \mathrm{C}$, which corresponds to $\alpha=2.064$ in Eq. (15).

We start our spinup experiment from a free atmosphere at rest. In the barotropic mode (Fig. 2a), an eastward jet forms above the oceanic front $(x>40)$ and two gyres appear on either side of the jet, as in F04: cyclonic to the north and anticyclonic to the south. A weaker circulation of opposite sign is observed to the west $(x<40$ : see the snapshot at $t=8$ nondimensional units in Fig. 2a). At this early stage in the spinup, the jet terminates fairly abruptly, at $x \sim 70=3500 \mathrm{~km} / L$, with a zone of diffluence where it splits into a return flow. Later on, the jet and the gyres spread eastward because of advection of the momentum by the jet itself, while the opposite circulation to the west weakens and later disappears (see $t=16$ and $t=120$ in Fig. 2a). The length of the atmospheric jet is now of roughly 4000 $\mathrm{km} / L$, more than 6 times the length of the oceanic front (see $t=120$ ). For orientation, $T_{i}=0.58$ day (see Table 1 ), so that $t=10,100,1000$ correspond roughly to 6,60 , and 600 days.

Later on still, at $t=136$, the jet and the two gyres break into two isolated dipoles, with a scale of $40=$ $2000 \mathrm{~km} / L$. Subsequently, the flow field becomes chaotic and eddies fill the domain $(t=344,1896)$. Most often, eddies detach from the meanders downstream, along the eastern most portion of the jet; see, for instance, $t=2544$. The detached anticyclonic eddies move northward along the eastern tip of the cyclonic gyre $(t=2552)$, while the cyclonic eddies move southward along the eastern tip of the anticyclonic gyre. These eddies do not seem to penetrate the large gyres themselves, which would eventually weaken their vorticity; instead, they are themselves weakened and washed upstream by the flow on the periphery of the main gyres. During the integration time, there are long intervals, of 20-60 days, when the barotropic flow field resembles the one at $t=4304$, with a large dipole and a fairly long jet of $50=2500 \mathrm{~km} / L$ (about 4 times the length of the oceanic front).

The time evolution of the baroclinic mode is shown in Fig. 2b. A long eastward jet forms above the oceanic front and further to the west $(x<60)$; it is associated with two gyres, one on either side of the jet. A dipole with a cyclonic vortex to the south and an anticyclonic vortex to the north of the symmetry axis forms to the east of the jet $(x>60)$ and propagates eastward. This propagation, as seen between $t=8$ and 16 , is probably 


\section{(a) Barotropic mode}
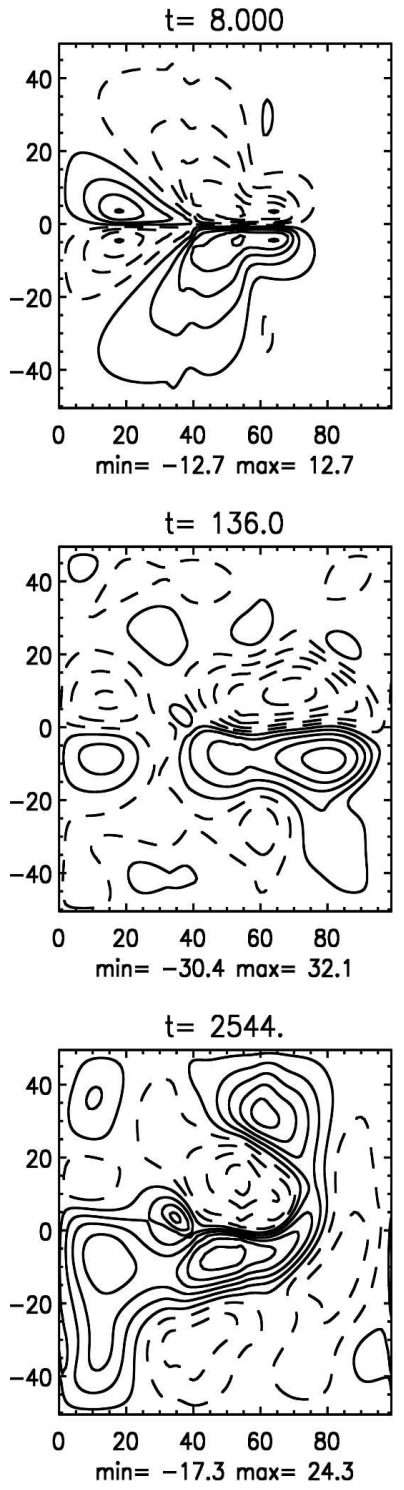
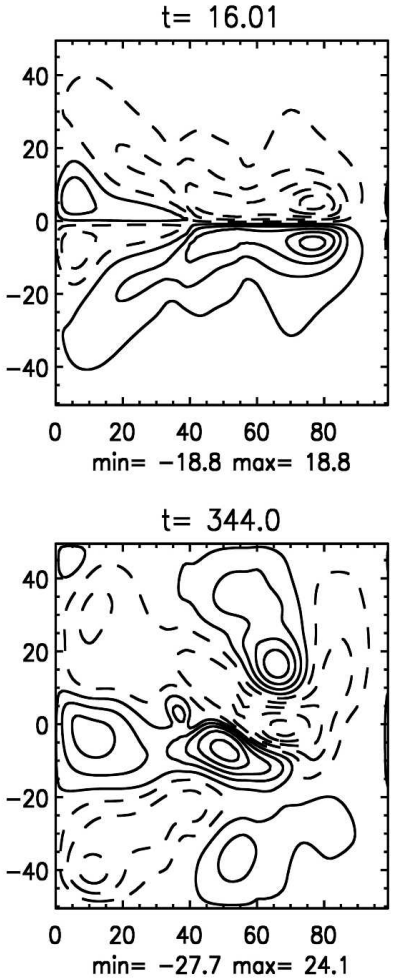

$\mathrm{t}=2552$.

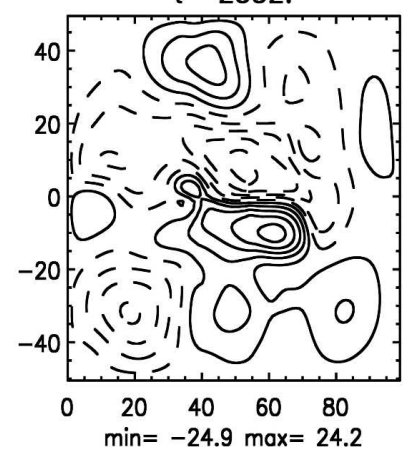

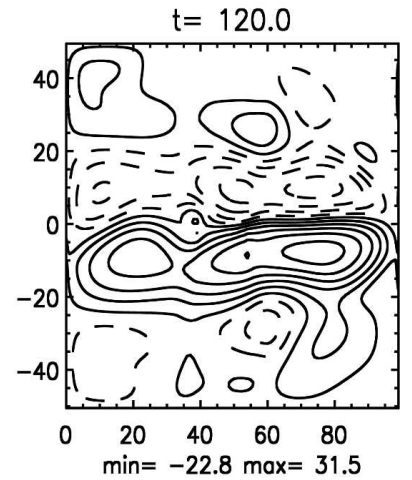
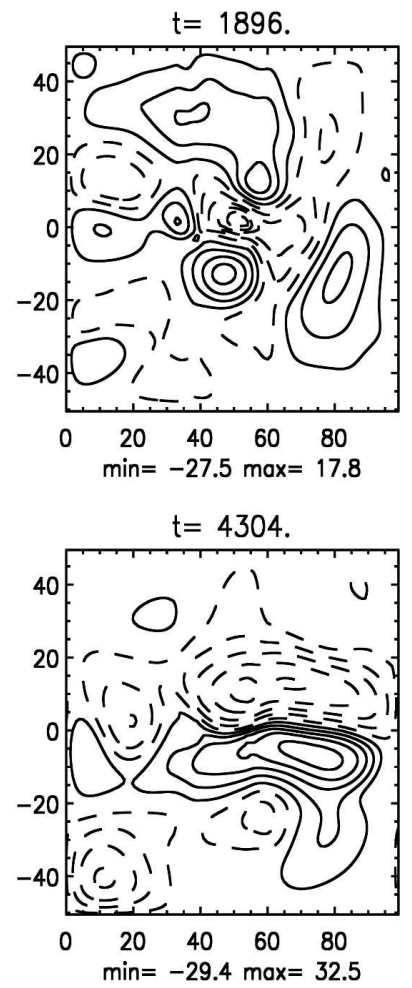

FIG. 2. Spinup experiment for the SST front of Fig. 1. Evolution of the (a) barotropic and (b) baroclinic mode. All spatial patterns are shown for the same rectangular domain of $5000 / L \mathrm{~km} \times 5000 \mathrm{~km} / L$. The scaling of the axes in this figure and in all the subsequent spatial plots is in nondimensional units; these units have to be multiplied by $50 \mathrm{~km}$ to yield dimensional distances. Snapshots shown at unequally spaced time in (a), (b); each plot has 10 contour intervals: $\mathrm{CI}=(\max -\min ) / 10$, where the $\max$ and $\min$ values are given in the legend of each plot.

due to the advection by the barotropic flow, which has the opposite polarity, in this part of the domain and at this time.

Later on, at $t=120-136$, the dipole downstream of the SST front grows and becomes the dominant feature. Still later, the flow becomes turbulent and the domain is filled with eddies (see Fig. $2 \mathrm{~b}$ at $t=344,1896$ ). The baroclinic component of the circulation is roughly op- posite to the barotropic one, which is still dominant. The total flow (not shown) is baroclinic, with an eastward jet and eddies that are stronger in the upper layers; see the range of the values for the nondimensional streamfunction in Figs. 2a,b, respectively. In fact, the ratio between the amplitude of the baroclinic and barotropic modes changes during the spinup stage. At the beginning (not shown), this ratio is larger than one, 


\section{(b) Baroclinic mode}
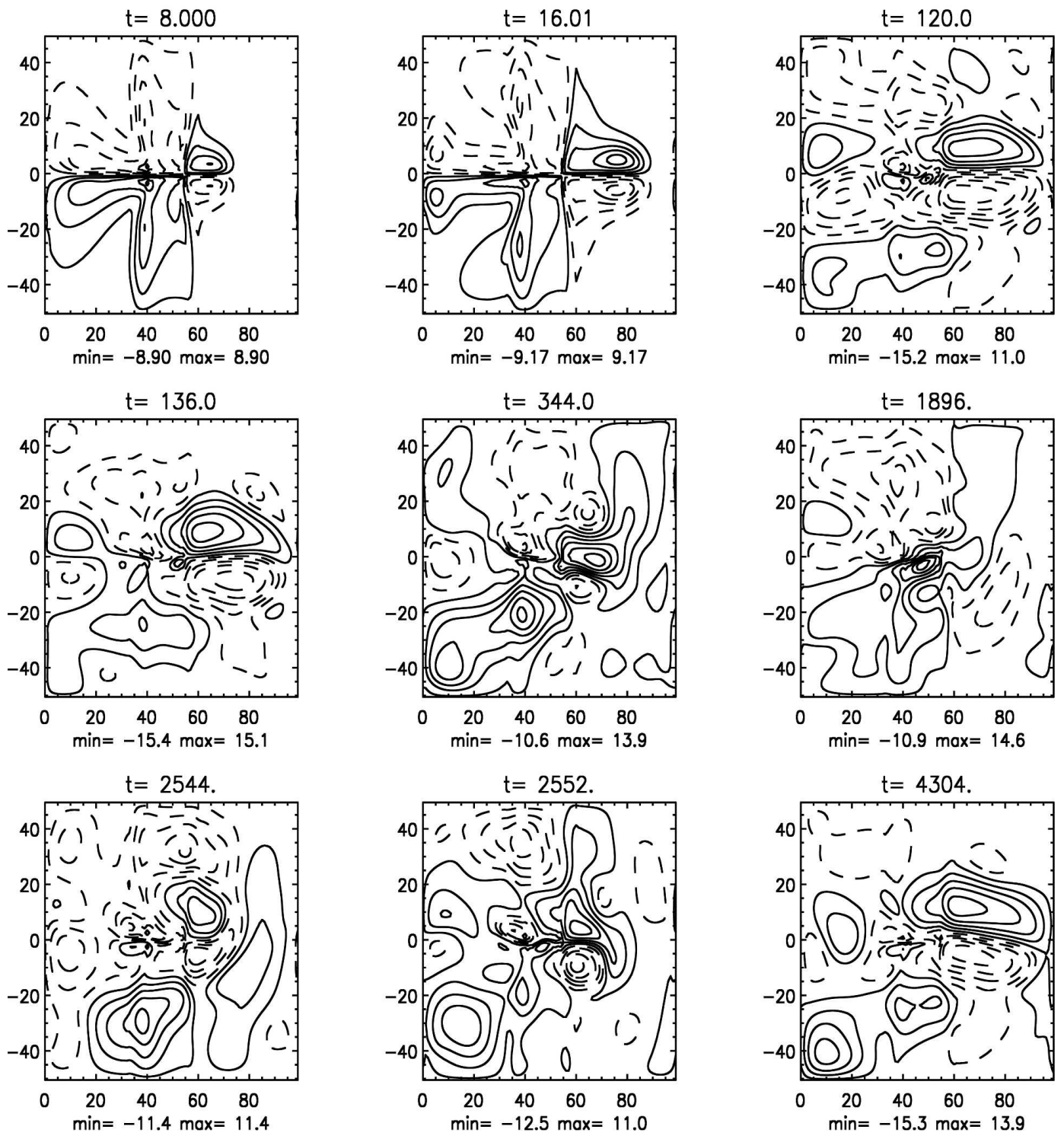

then, at $t=8$, it decreases to 0.7 , and still later, at $t=$ 16 , this ratio decreases to 0.5 and remains stable.

\section{b. Oscillatory components of the flow field}

The model is integrated for $8.2 \mathrm{yr}$ (5150 nondimensional time units) until it reaches a statistical equilibrium. We discard the initial segment of roughly $2 \mathrm{yr}$, in order to eliminate the transients, and we examine the time series of the last $6.2 \mathrm{yr}$. In this stationary regime, the solution is aperiodic in time and asymmetric in space. Every 500 time steps, that is, every 4.63 days, we save the streamfunction and PV field over that sampling interval.

The mean of the streamfunction field over the last 6 yr of the simulation is shown in Fig. 3. The mean barotropic streamfunction (Fig. 3a) exhibits two antisymmetric gyres - cyclonic north of the SST front and anticyclonic to the south-separated by a purely zonal jet. The western edge of the core of the two gyres lies at the same longitudinal location as the western edge of the SST front (cf. Fig. 3 and Fig. 1), while their eastern edge lies considerably downstream of the front's eastern 

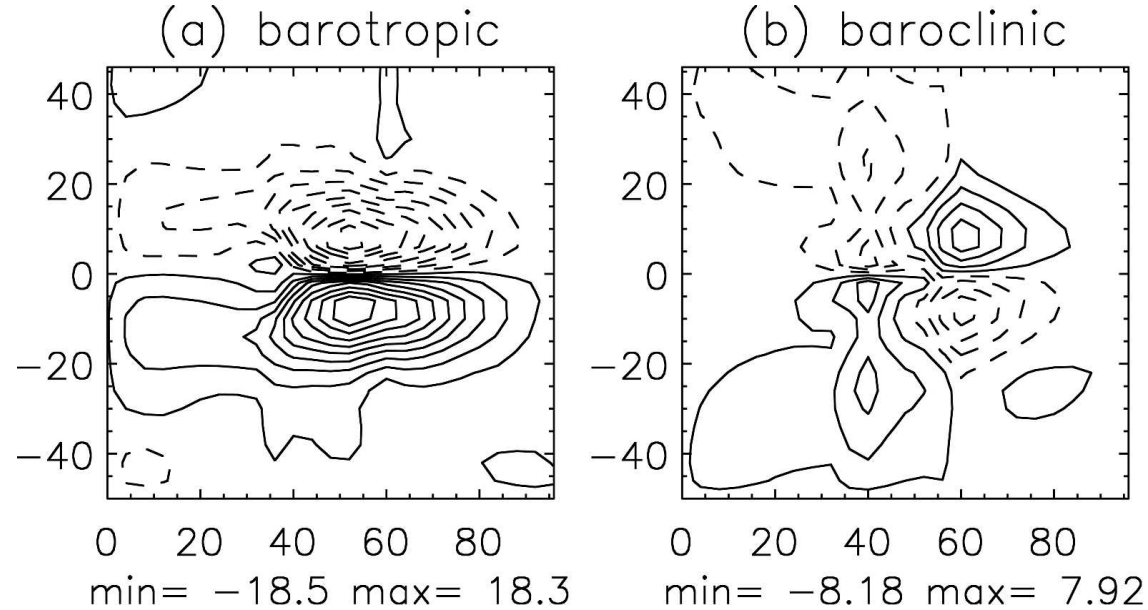

FIG. 3. Mean streamfunction field for $T^{*}=6.1^{\circ} \mathrm{C}(\mathrm{CI}=2)$ : (a) barotropic and (b) baroclinic.

edge. The mean baroclinic streamfunction (Fig. 3b) over the SST front itself is associated with two opposite gyres, cyclonic to the north and anticyclonic to the south, while downstream of the front $(x>60)$ the baroclinic flow exhibits two gyres with the opposite vorticity; that is, anticyclonic to the north and cyclonic to south. The total circulation, because of the superposition of the barotropic and baroclinic streamfunctions, induces a jet that is both stronger and longer in the upper layer than in the lower (not shown).

The power spectrum of the mean kinetic energy, defined over the integration domain, is shown in Fig. 4. We used the multitaper method (Thomson 1982; Mann and Lees 1996) with three tapers and bandwidth parameter 2, as well as other methods in the Singular Spectrum Analyses-Multitaper Method (SSA-MTM)

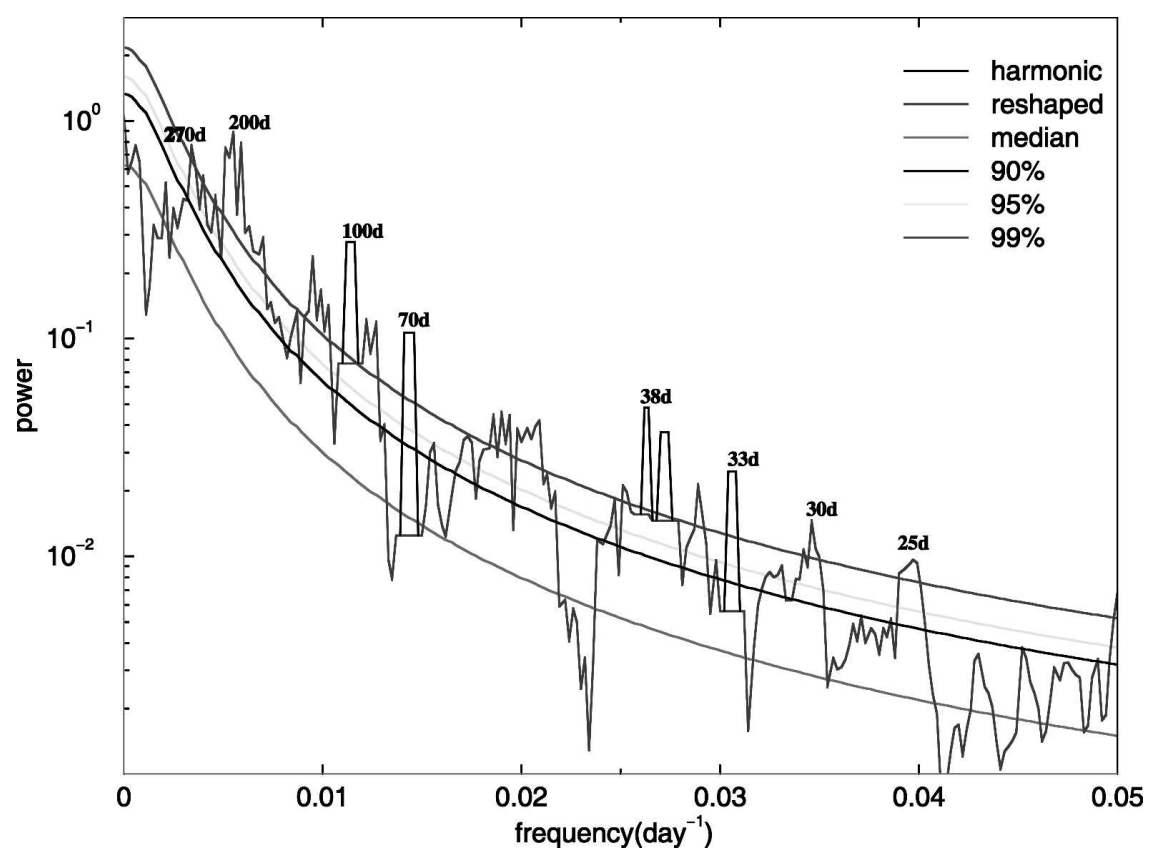

FIG. 4. Power spectra of the total kinetic energy; this and subsequent Figs. 5-9 refer to the control experiment with $T^{*}=6.1^{\circ} \mathrm{C}$ and $\xi=-1$. The spectra were calculated using the MTM with three tapers and a bandwidth parameter of 2. Following Mann and Lees (1996), several types of spectra (harmonic, reshaped, and median; see legend) were calculated and the labeled peaks all exceed the $99 \%$ confidence level. 
Toolkit (Ghil et al. 2002; http://www.atmos.ucla.edu/ $\mathrm{tcd} / \mathrm{ssa}$ ). The most prominent peaks that are common to the results of the various spectral methods lie near 270 , $100,70,33$, and 25 days. These results are fairly stable, given the small ratio of the significant periods to the length of the simulation, and the ones cited have all a statistical significance of at least $95 \%$ and, in certain cases, of more than $99 \%$.

We next identified the coherent spatiotemporal patterns in the barotropic and baroclinic streamfunction fields, which are associated with the spectral peaks found in the kinetic energy. To do so, we performed a combined multichannel singular-spectrum analysis (MSSA; see Keppenne and Ghil 1993; Plaut and Vautard 1994; Ghil et al. 2002) of these two fields. To reduce the size, and thus improve the condition number, of the covariance matrices involved in the M-SSA calculations, we sampled the two fields being analyzed every four grid points along each axis and performed principal component $(\mathrm{PC})$ analysis in space on the $25 \times 25$ grid so obtained. The 10 leading empirical orthogonal functions (EOFs) capture about $95 \%$ of the total variance of the subsampled field, including both the barotropic and baroclinic modes. We then carried out the joint M-SSA on the 10 associated PCs with a window width of 80 sampling intervals, that is, of about 370 days. This window width allows us to analyze all the periodicities marked in Fig. 4. The composites of each periodic mode were computed, in eight phase categories, according to the methodology of Moron et al. (1998, see their appendix for details).

The leading EOF pair of our M-SSA analysis corresponds to the 270-day peak in Fig. 4. The composite anomalies of this oscillatory mode are shown in Fig. 5. The anomalies are mainly antisymmetric with respect to $y=0$, and correspond to a standing dipole whose intensity oscillates between two extremes of opposite signs. The centers of the two gyres are located at $x \simeq 70$ in both the barotropic (Fig. 5a) and the baroclinic (Fig. $5 b)$ modes. The amplitude of the baroclinic mode in this oscillatory pair is half the amplitude of the barotropic mode and the two modes have opposite signs at all times.

The 105-day oscillation extracted by M-SSA has a similar antisymmetric pattern as the 270-day oscillation; it is propagating northward, however, as shown in Fig. 6. This northward propagation is the result of an interference phenomenon between a standing oscillatory pattern that is antisymmetric and a symmetric oscillatory one with roughly the same period. To describe this interference, we applied M-SSA separately to the symmetric (S) and antisymmetric (A) parts, $\psi_{S}$ and $\psi_{A}$, of the streamfunction field:

$$
\begin{aligned}
\psi_{S} & =\frac{1}{2}[\psi(y)+\psi(-y)], \\
\psi_{A} & =\frac{1}{2}[\psi(y)-\psi(-y)] .
\end{aligned}
$$

By using this decomposition, we were able to isolate two oscillatory modes (not shown), one symmetric and propagating slightly westward, the other standing and antisymmetric; both have a period of 105 days and yield by superposition the 105-day northward-propagating mode of Fig. 6.

The northward-propagating 105-day mode here could be related to the observed 70-day oscillation that propagates from Cape Hatteras to Greenland in a direction roughly perpendicular to the Gulf Stream path (see Plaut and Vautard 1994). In fact, the relative variances of the two oscillations, in the observations and in the model, are quite similar: Plaut and Vautard (1994) found it to be $1.6 \%$ of the total or $2.7 \%$ after removal of the annual cycle, while it is of $2.4 \%$ in the model, which has no seasonal cycle.

The reconstructed 30-day oscillation is shown in Fig. 7. The barotropic streamfunction anomaly (Fig. 7a) is mainly symmetric with respect to $y=0$. This anomaly begins to develop at $x \simeq 85$ (the negative anomaly at phase $t=3.75$ ) and then propagates westward, while becoming stronger and more elongated along the $x$ axis ( $t=7.5-11.2$ days). Later, it decays in place $(t=15$ days), before changing sign $(t=18.7$ days $)$. The baroclinic streamfunction anomaly (Fig. 7b) has still half the amplitude of the barotropic one and the opposite sign, like in the 270-day oscillation. The equivalentbarotropic structure of this symmetric oscillation is very similar to the 30-day instability found by F04 in their equivalent-barotropic model (see Fig. 9 and section $4 \mathrm{~b}$ there). We thus conclude that two distinct types of oscillatory modes coexist in our baroclinic model: standing, antisymmetric dipoles and symmetric modes that have a slight tendency to travel, or expand, westward.

To gain further insight into the dynamics of these two types of modes, we use the symmetric-antisymmetric decomposition Eq. (16) and analyze the two components separately. The dominant oscillations in each component are the same as those obtained in the previous M-SSA analysis.

\section{c. Energy evolution}

The kinetic energy of the barotropic and baroclinic mode of the flow is shown in Fig. 8. We identify two energy states: a high-energy state, in which the energy of the barotropic mode is larger than 0.9 nondimensional units; and a low-energy state, in which it is lower than 0.6. The flow in the high-energy state (not shown) 


\section{(a) Barotropic mode}
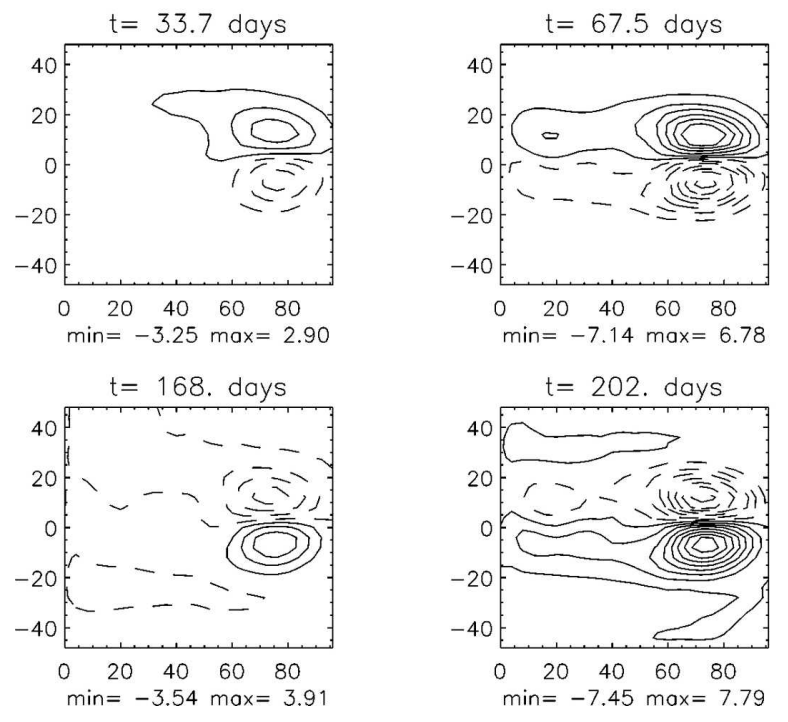

\section{(b) Baroclinic mode}
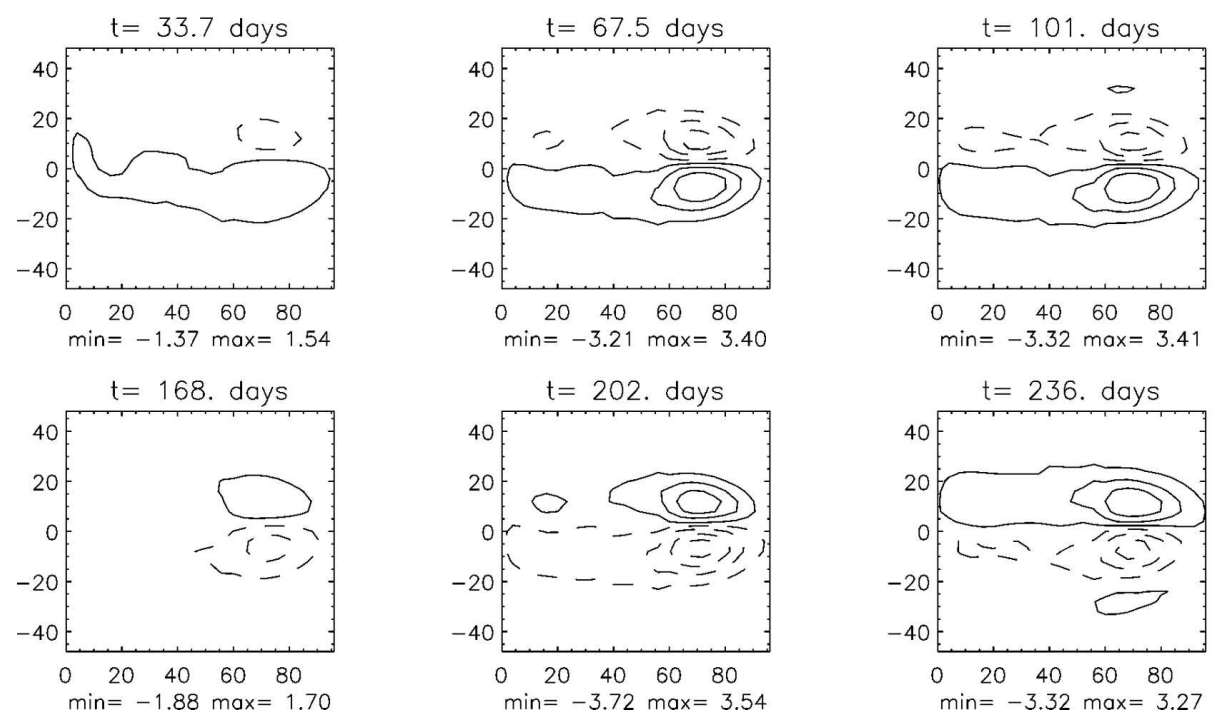
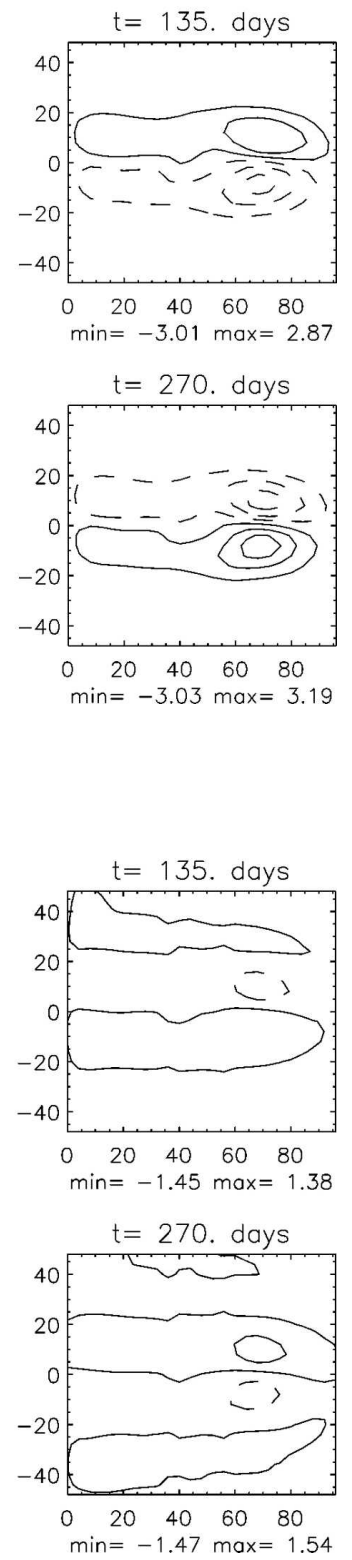

FIG. 5. Reconstructed 270-day oscillation, based on pair 1-2 of our M-SSA; this pair captures $6 \%$ of the total variance. (a) Barotropic and (b) baroclinic mode; phase 1 of the 8 phase composites is in the upper-left panel (CI $=1$ in nondimensional units) in both modes.

is very similar to that found at $t=136,4304$ in Fig. 2a; that is, two large gyres occupy the eastern part of the domain and are associated with a strong eastward jet. The flow in the low-energy state (not shown) is characterized by a short jet, mostly located over the SST front, and two small gyres surrounded by several eddies, similar to the situation at $t=1896,2544$ of Fig. 2b.

The energy of the barotropic mode is about 3 times larger, on average, over the entire simulation, than the baroclinic one. This result is due to the energy cascade from the baroclinic to the barotropic flow component, as shown in Fig. 9. Indeed, the energy transfer term between the baroclinic and barotropic mode $e_{10}$ in Fig. 9 dissipates the energy in the baroclinic mode [see Eq. (13a) for $k=1$ ] whereas the energy transfer term $e_{01}=$ $-e_{10}$ gives energy to the barotropic mode. Note that other processes play a role as indicated later at the end of this section. 

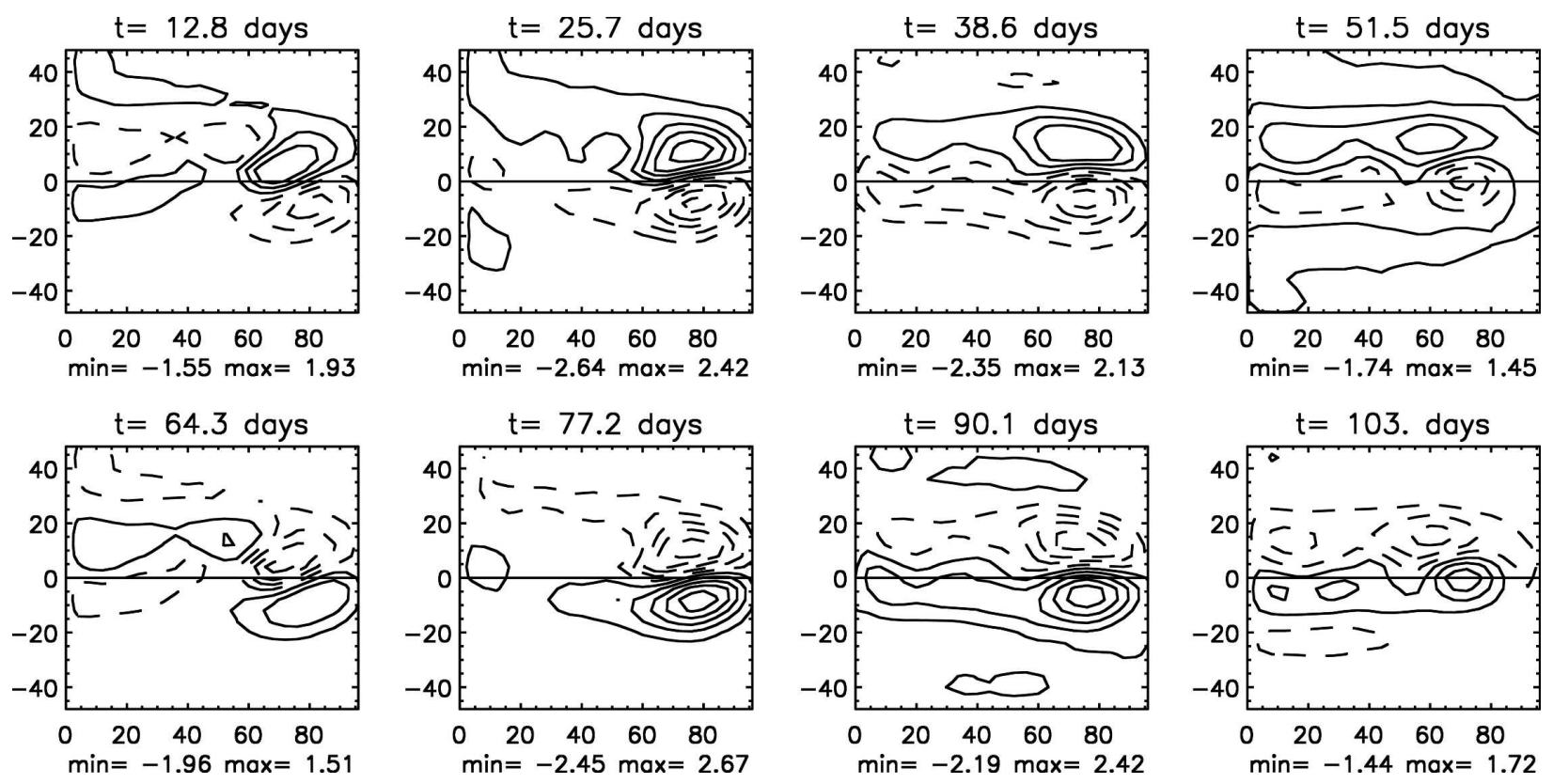

FIG. 6. Barotropic mode of the reconstructed 105-day oscillation, based on pair 10-11; this pair captures $2.4 \%$ of the total variance $(\mathrm{CI}=0.5$ nondimensional units). Same ordering of phases as in Fig. 5.

At the beginning of the spinup process (Fig. 2), the energy in the baroclinic mode is larger than in the barotropic mode (not shown). The jet at this stage is confined to a thin layer above the AMBL, as in Doyle and Warner's (1990) simulation of Genesis of Atlantic Lows Experiment (GALE) observations, for the first $12 \mathrm{~h}$ of their mesoscale model integration. The transition from this early stage of a spinup simulation to the asymptotic, statistically stationary regime studied in sections $3 \mathrm{~b}$ and $3 c$ occurs through the energy transfers illustrated in Fig. 9.

The energy cascade toward larger total scales, horizontal as well as vertical, is a major feature of QG turbulence (Charney 1971) in the presence of the $\beta$ effect (Rhines 1975). Horizontal scales larger than the Rossby radius of deformation are associated with an energy cascade from the baroclinic to the barotropic mode (Fu and Flierl 1980; Salmon 1998). The energy injection from the oceanic front through the AMBL into the baroclinic mode is about 1.3 times larger than the injection into the barotropic mode (see Fig. 9). We thus conclude that the large amount of energy found in the barotropic mode is due to this cascade to lower vertical wavenumber: the AMBL provides more energy into the baroclinic mode, which then cascades into the barotropic mode.

Examining further the energy transfer between the A and S components [see Eq. (16)], we can identify two kinds of instabilities, barotropic and baroclinic. The time series of the barotropic kinetic energy and of both its $\mathrm{A}$ and $\mathrm{S}$ components is shown in Fig. 10. The energy growth in the S component and decrease in the A component is due to the energy transfer from $\mathrm{A}$ to $\mathrm{S}$. The barotropic instability is revealed by the energy transfer from the SST front through the AMBL to the A component and then to the perturbation $\mathrm{S}$ component. This barotropic-instability-related transfer is most prominent over relatively short time intervals, of about 30 days.

The energy transfer from the baroclinic to the barotropic mode indicates that baroclinic instabilities are also present, as shown in Fig. 9. As shown later (end of this section), these baroclinic instabilities are associated to antisymmetric unstable modes with periods longer than 100 days.

The energy in the barotropic mode's A component is larger than the energy in its S component at all times. The high-energy states correspond to flows that are characterized by a strong, persistent dipole; that is, the dominant, barotropic flow component is nearly antisymmetric (see Fig. 2a at $t=4304$ ). The high-energy episodes last about 200-300 days. The A and S components are almost anticorrelated, with the minima of the latter preceding slightly the maxima of the former, and vice versa.

The energy exchanges between the A and S components of the solution can be explained as follows. First, we note that the A component is dominated by the jet, while the $\mathrm{S}$ component captures perturbations away from it. The atmospheric jet induced by the SST front is 


\section{(a) Barotropic mode}
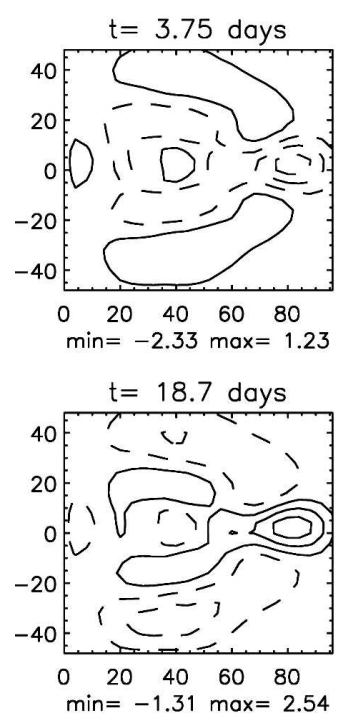
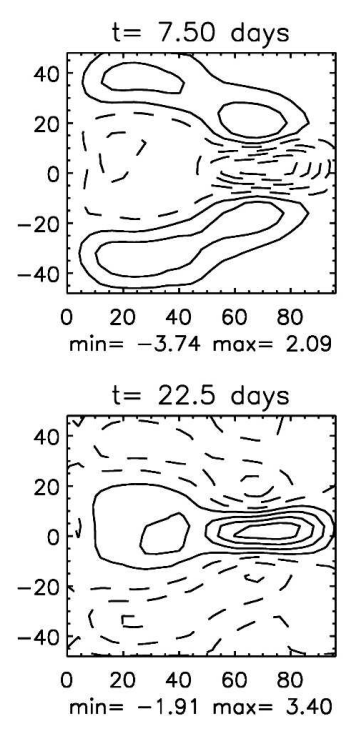

\section{(b) Baroclinic mode}
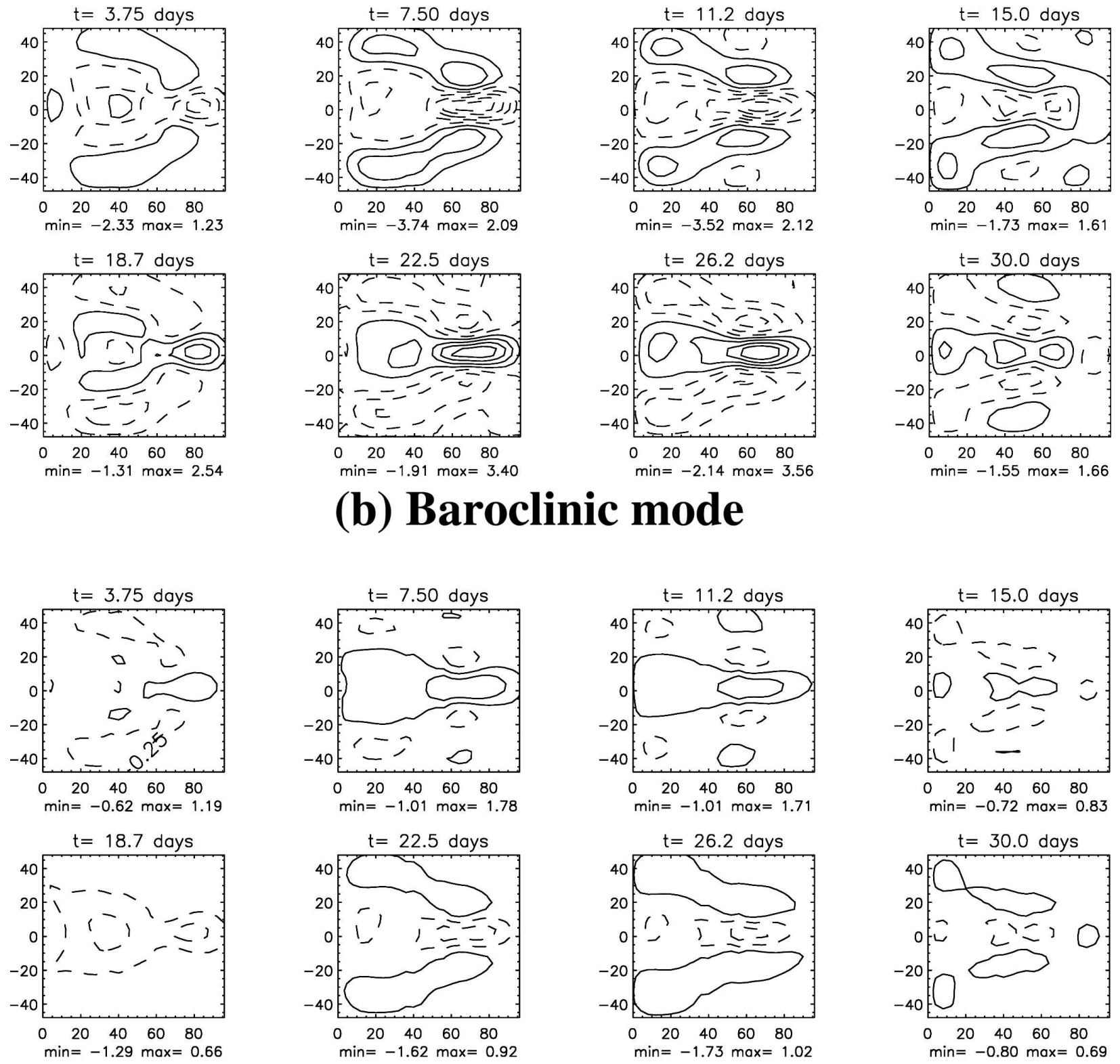

FIG. 7. Reconstructed 30-day oscillation, based on pair 4-5 of our M-SSA; this pair captures $4.6 \%$ of the total variance. (a) Barotropic and (b) baroclinic mode $(\mathrm{CI}=0.75$ in nondimensional units) in both modes.

destabilized by a symmetric barotropic instability (see F04) that enhances the S component of the flow and thus weakens its A component.

Since $T$, and hence $\nabla^{2} T$ is antisymmetric,

$$
\int_{\Omega} \alpha \psi_{S} \nabla^{2} T d \omega=0
$$

It follows from (17) that the SST front injects energy into the flow only via its A component $\psi_{A}$; see Eq. (13a). Therefore, as this component of the flow is weakened, there is less energy injection and so the jet is stabilized again. The nonlinear energy transfer to the $S$ component stops and the energy in this component reaches a local maximum, shortly after the energy of the A component has reached a local minimum.

The injection of energy from the lower boundary, via the A component of the flow, continues so that the energy in this component increases again, while the en- 


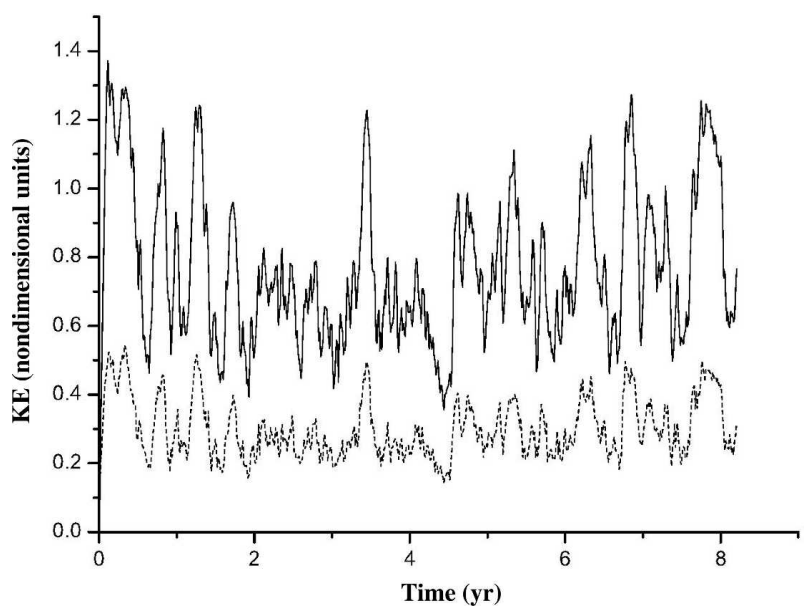

FIG. 8. Kinetic energy (KE) as a function of time: barotropic mode (solid line) and baroclinic mode (dashed line) of the kinetic energy.

ergy in the $\mathrm{S}$ component decreases due to friction and reaches a minimum. The strength of the A component and of the associated jet meanwhile increase, until the latter reaches its local maximum and becomes unstable. These opposite tendencies can be seen in Fig. 10a: the energy of the A component increases when the energy of the $\mathrm{S}$ component decreases and vice versa.

As noted already, the phase relations between the A and $\mathrm{S}$ components in Fig. 10a appear to be characteristic of barotropic instability. To highlight the processes associated with this instability, we focus now on the

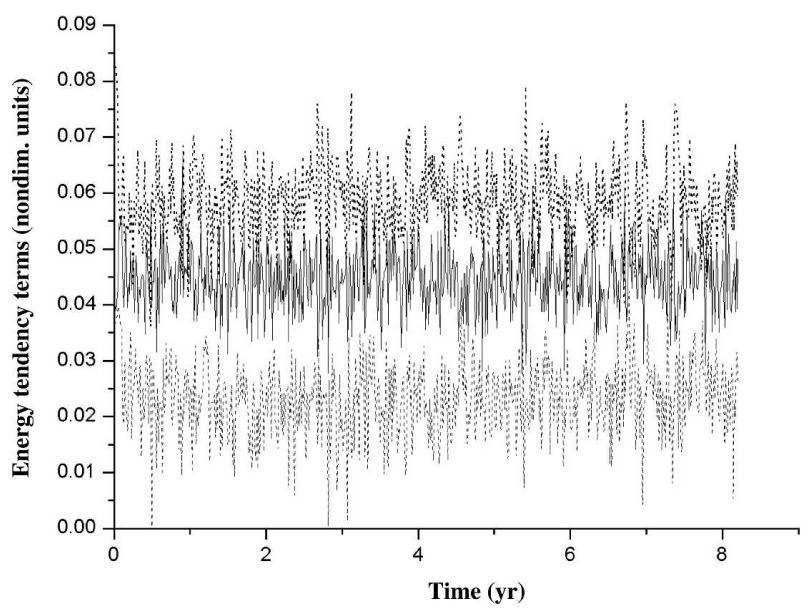

FIG. 9. Energy tendency terms as a function of time. Dotted line (lowermost curve): energy transfer term between the baroclinic and barotropic mode: see Eq. (13c), $i=0, k=1$. Solid line (middle curve): energy injection term $\psi_{0} \phi_{0}(0) w(z=0)$ from the AMBL into the barotropic mode. Dashed line (uppermost curve): energy injection term $\psi_{1} \phi_{1}(0) w(z=0)$ from the AMBL into the baroclinic mode. a) $\xi=-1, H_{2}=0.28$

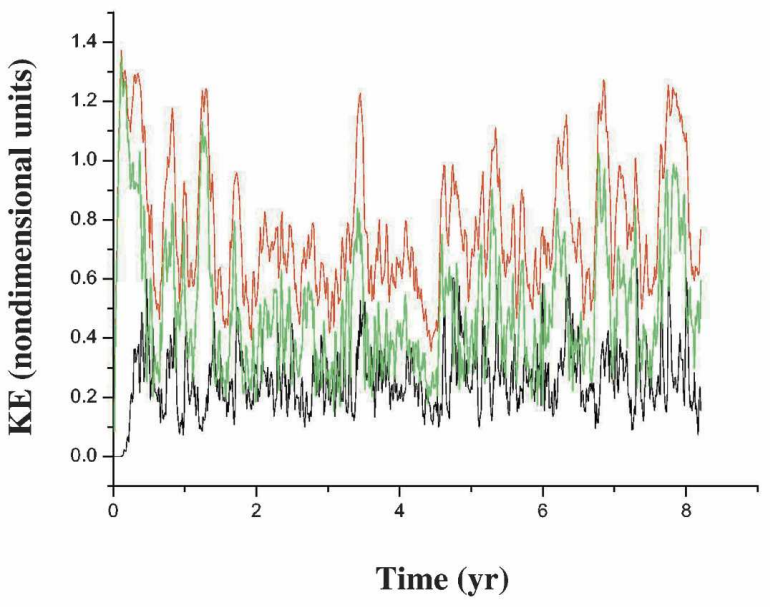

b) $\xi=-2.5, H_{2}=0.11$

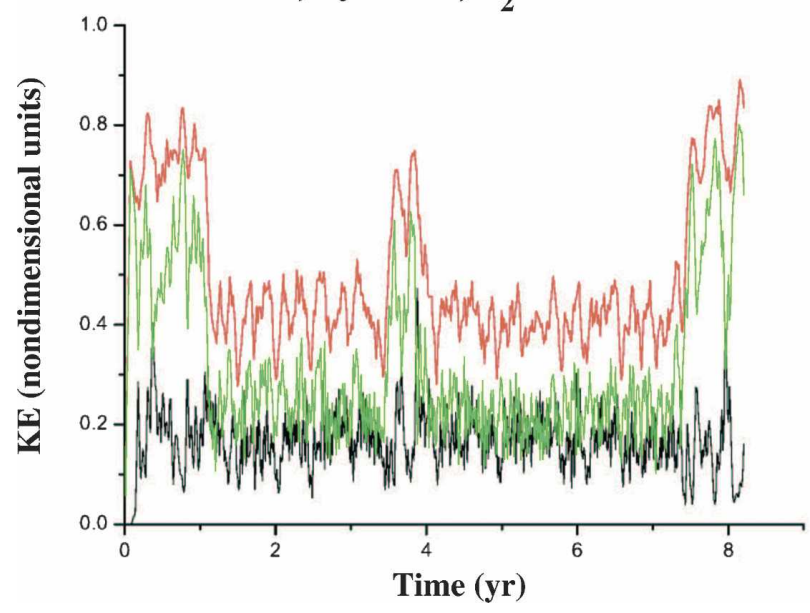

FIG. 10. Kinetic energy of the barotropic mode as a function of time, for $T^{*}=6.1^{\circ} \mathrm{C}$ : (a) $\xi=-1, \mathrm{H}_{2}=0.27$; (b) $\xi=-2.5, \mathrm{H}_{2}=$ 0.11 . Total kinetic energy of the mode in red, antisymmetric component $(\mathrm{A})$ in green, and symmetric component $(\mathrm{S})$ in blue.

30-day oscillation, which is, as we shall see, driven by it. Figure 11a shows the M-SSA reconstruction of the 30day oscillatory mode extracted from the kinetic energy time series. The $\mathrm{S}$ component (solid line) is in phase opposition and has the same amplitude as the A component (dotted line); this phase relationship is a key feature of the barotropic instability that gives rise to the westward-propagating, symmetric mode shown in Fig. 7.

The contribution of baroclinic instability to the full variability is most clearly seen in the 270 -day mode, illustrated in Fig. 11b. Here the A component (dotted) leads the $\mathrm{S}$ component (solid) by one quarter period. The A component is roughly 4 times larger than the $\mathrm{S}$ component and has to draw its energy from the baroclinic mode. This inference is supported by the energy transfer diagram shown in Fig. 12. Finally, the energet- 
(a) 30-day oscillation

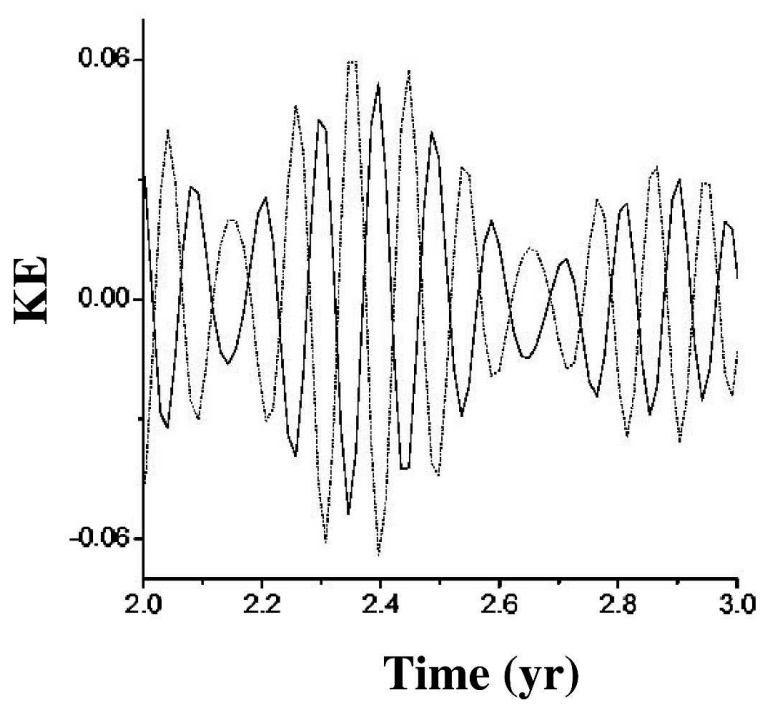

(b) 270-day oscillation

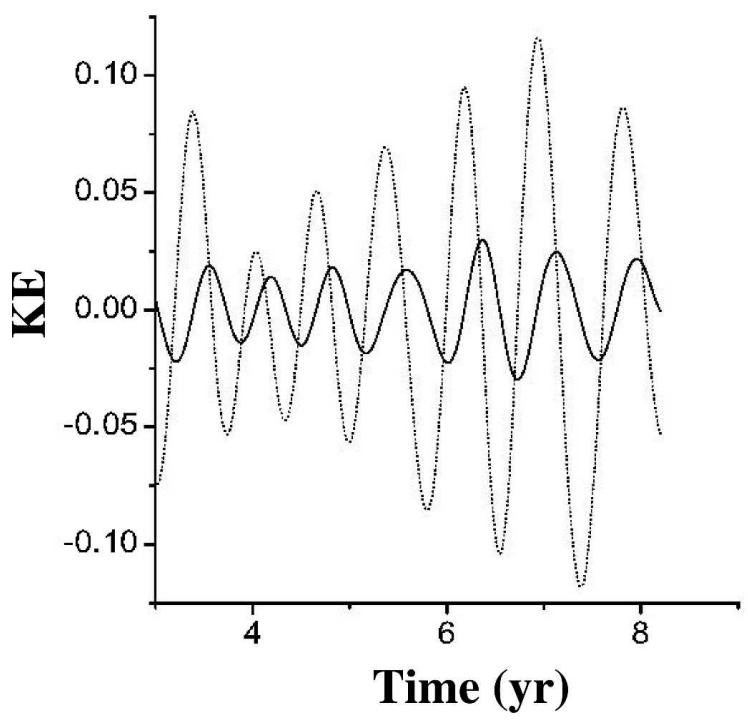

FIG. 11. M-SSA reconstruction of the oscillatory modes in kinetic energy for the control run $\left(T^{*}=6.1^{\circ} \mathrm{C}, \xi=-1\right)$ : (a) 30-day mode, during years 2 and 3; and (b) 270-day mode during years 3-8; A component (dotted line) and S component (solid line); the former leads the latter by one-half period in (a) and by onequarter period in (b).

ics of the 105-day mode (not shown) is driven by mixed, barotropic-baroclinic instability.

Figure 12 summarizes the different energy transfers between the $\mathrm{A}$ and $\mathrm{S}$ components of the barotropic and baroclinic modes, for different values of the layer-depth ratio $H_{2} / H_{1}$, and hence of the parameter $\xi$; the case of $\xi=-1$ studied so far appears in the middle panel. Note that our model represents an open system, with energy
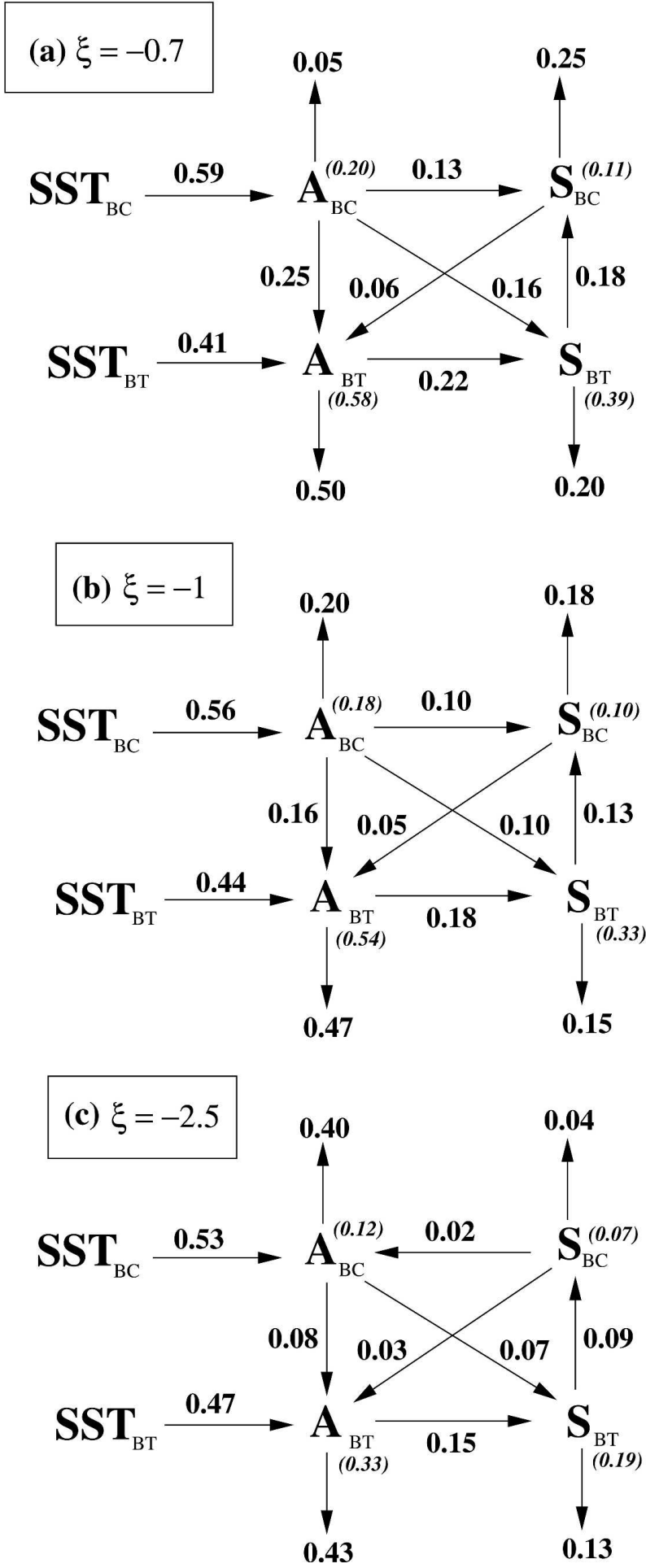

FIG. 12. Mean energy transfers between the antisymmetric (A) and symmetric (S) components of both barotropic (BT) and baroclinic (BC) modes for $T^{*}=6.1^{\circ} \mathrm{C}$ and different values of the parameter $\xi$; see Table 2 for the corresponding layer thicknesses. The energy transfer rates are indicated in bold for each arrow, while the nondimensional energy value of each mode and component is indicated in italics and in parentheses. 
input from the lower boundary and energy output through viscous dissipation. The input and output, however, are exactly equal to each other and normalized for simplicity to equal 1.0, while the energy densities are normalized by the same value of $V^{2}=1 \mathrm{~m}^{2} \mathrm{~s}^{-2}$; see Eq. (1) and Table 1.

From this figure, we conclude that (i) there is more energy injected into the baroclinic mode than into the barotropic mode; while (ii) the loss of energy due to dissipation is always larger from the barotropic mode than from the baroclinic mode, and these losses are much more sensitive to $H_{2} / H_{1}$. The dissipation of the flow's A component (both baroclinic and barotropic) is always stronger, while the loss from the baroclinic A component is most sensitive to the stratification $H_{2} / H_{1}$.

It is interesting to observe that the barotropic energy is dissipated at a slower rate than the baroclinic energy in every case. Dividing the energy in a mode by its rate of dissipation, we find that the barotropic time scale of dissipation is about twice as long as the baroclinic one; this makes the barotropic mode contain more energy than the baroclinic one. These differences in dissipation rates are superimposed on the basic energy cascade and modify the energy distribution.

\section{Sensitivity studies}

\section{a. Sensitivity to layer-depth ratio}

The behavior of the real atmosphere depends on the stratification $S=S(z)$ of the mean state. This state change from summer to winter, between warmer and cooler episodes within each season, as well as with latitude. In this subsection, we examine therefore the influence of the layer-depth ratio or, equivalently, the triple interaction coefficient $\xi$ on the dynamics; see Eq. (12).

In this study, the Rossby radius

$$
\frac{L}{\lambda_{1}}=\frac{\Delta \rho}{\rho_{0}} \frac{g H_{1}\left(H-H_{1}\right)}{f^{2} H}
$$

is kept constant and equal to $575 \mathrm{~km}$ (i.e., $\lambda_{1}=50 / 575$ $=0.087$; see Table 1$)$. Thus $\Delta \rho$ and the symmetric product $H_{1} H_{2}$ vary in an opposite way, since $f, g, H=10000$ $\mathrm{m}$ and $\rho_{0}$ are all constant: as $H_{1} H_{2}$ increases, $\Delta \rho$ decreases, and vice versa. The maximum of $H_{1} H_{2}$ occurs at $H_{1}=H_{2}=1 / 2$, where $\Delta \rho$ thus has its (nonzero) minimum. But, in fact, the overall problem is not as symmetric with respect to the layer thicknesses as it appears from only considering Eq. (18). Since the energy and PV are injected from the lower surface, the two extreme cases in which $\Delta \rho=\rho_{2}$ and either $\xi=-4.8$ and $H_{2}=0.04 H$ or $\xi=+4.8$ and $H_{1}=0.04 H$ are quite
TABLE 2. The effect of changes in the layer depths on flow characteristics. The first two columns give the parameter $\xi$ and the layer depths, the next two the periods of the most prominent oscillatory modes in the antisymmetric (A) and symmetric (S) components; the mean energy obtained in the BT and BC modes appears in the last column. Note that the lower layer is deepest for $\xi=-0.3$ and shallowest for $\xi=-2.5$.

\begin{tabular}{ccccc}
\hline \hline$\xi$ & $\begin{array}{c}H_{1}, H_{2} \\
(\mathrm{~m})\end{array}$ & $\begin{array}{c}\text { A component } \\
(\text { days })\end{array}$ & $\begin{array}{c}\text { S component } \\
(\text { days })\end{array}$ & $\begin{array}{c}\text { Mean energy } \\
(\mathrm{BT} / \mathrm{BC})\end{array}$ \\
\hline-0.3 & 5740,4260 & 71,114 & 33,71 & $1.26 / 0.40$ \\
-0.7 & 6650,3350 & 200,100 & 33,29 & $0.96 / 0.31$ \\
-1.0 & 7230,2770 & 270,105 & 30,33 & $0.87 / 0.28$ \\
-1.3 & 7725,2275 & 220,122 & 40,34 & $0.77 / 0.26$ \\
-2.5 & 8900,1100 & 109,91 & 25,49 & $0.53 / 0.18$ \\
\hline
\end{tabular}

different: the former case is highly baroclinic, with strong instabilities related to the shallowness of the lower layer, while the latter is almost barotropic.

To complicate matters further, the model's long-term behavior is affected by the vertical propagation of instabilities and by the energy input and loss processes shown in Fig. 12. Because of these processes, the strong baroclinic instabilities associated with shallow $\mathrm{H}_{2}$ die out and the model settles into nearly barotropic dynamics, while such instabilities are entirely absent for sufficiently shallow $H_{1}$. The baroclinic transients become more and more persistent, though, as $H_{2} / H_{1} \rightarrow 1$, since the total losses from the baroclinic mode decrease from Fig. 12c to Fig. 12a. Hence the long-term behavior is most baroclinic for $H_{1}=H_{2}=1 / 2$, although the ratio of mean barotropic to mean baroclinic energy is roughly 3:1 (see Table 2), independently of layer-depth ratio.

It follows from the discussion thus far that the most interesting model behavior occurs when the lower layer is shallower, up to and including a case where it almost equals the upper layer in depth. The values of the lower-layer depth $H_{2}$ in Table 2 range from $H_{2}=1100$ $\mathrm{m}$ for $\xi=-2.5$ to $H_{2}=4260 \mathrm{~m}$ for $\xi=-0.3$.

For the values of $\xi$ shown in Fig. 12, we performed M-SSA on each flow component, symmetric (S) and antisymmetric (A), using the same values for the other parameters as before. In Table 2, the two strongest oscillatory modes are shown, separately for the A and S component, as a function of the ratio $H_{1}: H_{2}$ of upperto-lower-layer depth, given by $\xi$. The dominant period of the A component increases as the upper-layer depth $H_{1}$ decreases from $\xi=-0.3$ to $\xi=-1.0$ and reaches its maximum of 270 days for $\xi=-1$, only to decrease again when $\xi$ becomes more negative. For $\xi=-2.5$, the antisymmetric oscillatory modes are very weak. In the $S$ component, there is a dominant period of about one month for all values of $\xi$. Much longer periods are also found for all values of $\xi$. The periods of one and two 
months in the $\mathrm{S}$ component here are fully consistent with those obtained by F04 for an equivalentbarotropic atmosphere, in which all the instabilities were purely symmetric.

The mean energy, over the integration domain and time, as a function of $\xi$ is given in the last column of Table 2. As $\xi$ decreases in absolute value and tends to 0 ( $H_{2}$ increases $)$, the energy increases in both the baroclinic and barotropic mode, although the injection of vorticity from the AMBL into the free atmosphere (not shown) is the same for all the cases listed in Table 2. This PV injection is only a function of the oceanic front's intensity and not a function of $\xi$; see Eqs. (5) and (10). For the same amount of vorticity injected into the free atmosphere through its lower boundary, however, more energy is injected when the amplitude of the streamfunction is larger; this fact can be derived from the energy Eq. (13) and the boundary condition (5).

We examine below the change in the energy transfers for the three cases illustrated in Fig. 12, namely $\xi=$ $-0.7, \xi=-1$ and $\xi=-2.5$. The A component of the baroclinic mode becomes much more dissipated as the lower layer becomes shallower, whereas there is less transfer of energy into its $\mathrm{S}$ component. The baroclinic mode is thus overall less energetic when the lower layer becomes shallower and the energy injection from the SST front into it is thus reduced; this effect is noticeable in the weaker energy flow into the baroclinic mode in Fig. 12c and the significantly lower mean energy in this mode in the last row of Table 2 . This weakening of the baroclinic mode results in its providing less energy to the barotropic mode at lower $\mathrm{H}_{2}$ values.

The stronger flow for a deeper lower layer is associated with a more intense energy cascade from the baroclinic to the barotropic mode and thus a stronger barotropic flow (see the top two rows of Table 2 and Fig. 12a). The more intense barotropic mode, in turn, results in its receiving more energy from the AMBL (see Fig. 12a). This argument holds for all five cases examined in Table 2 and explains the fact that the mean energy increases as $\xi$ tends to 0 , that is, as $H_{2} / H_{1} \rightarrow 1$.

In Fig. 10b, the total energy of the barotropic mode and the energy of its $\mathrm{A}$ and $\mathrm{S}$ components are shown for the case $\xi=-2.5, H_{2} H=1100 \mathrm{~m}$. The high- and the low-energy states alternate, as in Fig. 10a, but the residence time in each state is longer, and the energy level more persistent: there are only three occurrences of a high-energy state, in the beginning, the middle, and at the end of the integration. The A component in the high-energy states is much larger, while the S component stays relatively constant throughout the simulation. The high- and low-energy states thus coincide with the high- and low-energy states of the A component.
As the lower layer becomes deeper, the frequency of occurrence of the high-energy states increases and their durations decrease, as can be seen by comparing Figs. 10a and 10b. The high-energy states in Fig. 10a have an even stronger downstream dipole and elongated eastward jet (not shown) than those associated with such states in Fig. 10b.

\section{b. Sensitivity to the oceanic front's intensity}

We now study the model dynamics as a function of the corrected frontal strength $T^{*}$ or, equivalently, of the parameter $\alpha$; the latter determines the strength of the vertical velocity at the top of the AMBL, according to Eq. (6). While varying $T^{*}$ in Eq. (14), we keep the frontal width $d=1=50 \mathrm{~km} / L$ fixed in Eq. (15). In this subsection, we study the oscillatory modes of the streamfunction by splitting it into its $\mathrm{A}$ and $\mathrm{S}$ components, as in Eq. (16), and analyze each component separately through M-SSA, with the same spectral parameters as in section $3 \mathrm{~b}$.

For $T^{*}=1.5^{\circ} \mathrm{C}$, the solution in the free atmosphere is steady, as it was in F04. The barotropic mode has two antisymmetric gyres - cyclonic to the north of the front and anticyclonic to the south-separated by a purely zonal jet (see Fig. 13). The circulation in this case is similar to the mean circulation shown in Fig. 3, but with a much smaller strength and horizontal scale.

For $T^{*}=3^{\circ} \mathrm{C}$, the solution is already chaotic; in the equivalent-barotropic model of F04, the first Hopf bifurcation to periodic solutions occurred at $T^{*}=4^{\circ} \mathrm{C}$, while transition to irregular flows only arose at $T^{*} \simeq$ $5^{\circ} \mathrm{C}$. The two dominant oscillatory modes of the $\mathrm{S}$ component have periods of 22 and 44 days, while the $\mathrm{A}$ component has dominant periods of 200 and 85 days. The spatial patterns of the oscillatory modes of each component (not shown) are very similar to those found for $T^{*}=6.1^{\circ} \mathrm{C}$, but with smaller scales and strength; see Figs. 7 and 5, respectively.

In Table 3, the period of the two dominant oscillatory modes of the $\mathrm{A}$ and $\mathrm{S}$ components are given as a function of the strength $T^{*}$ of the SST front or, equivalently, as a function of $\alpha$. The dominant periods of the A component are much larger than in the $\mathrm{S}$ component. In the latter, we also find very low-frequency variability by applying a moving-average filter of 10 time intervals (46.3 days). The two dominant oscillations found in this filtered time series are also shown in Table 3. For $3^{\circ} \mathrm{C} \leq T^{*} \leq 9.1^{\circ} \mathrm{C}$, the longest period so obtained increases, from two to about six months, and it stabilizes afterwards.

In Fig. 14, we plot the period of the dominant modes versus the thermal front's strength $T^{*}$. For $3^{\circ} \mathrm{C} \leq T^{*} \leq$ $6.1^{\circ} \mathrm{C}$, the dominant period in the $\mathrm{A}$ component in- 
(a) Barotropic

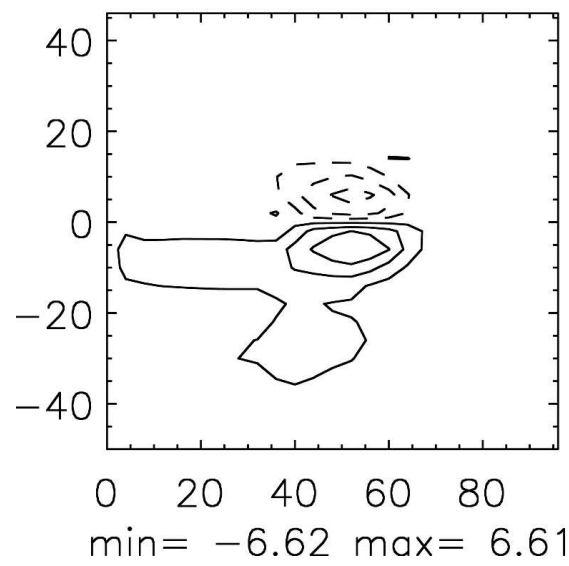

(b) Baroclinic

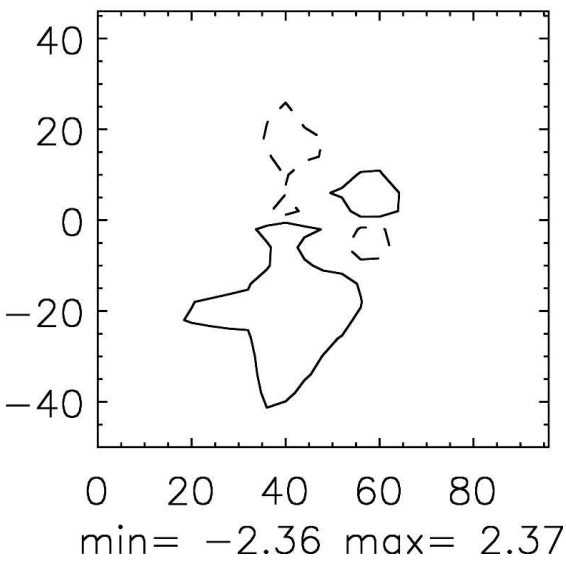

FIG. 13. Steady-state solution for $T^{*}=1.5^{\circ} \mathrm{C}$ and $\xi=-1$ : (a) barotropic and (b) baroclinic streamfunction field, $\mathrm{CI}=2$ nondimensional units.

creases as $T^{*}$ increases; it reaches its maximum at $T^{*}=$ $6.1^{\circ} \mathrm{C}$ and then decreases. For the $\mathrm{S}$ component, the dominant period in the raw series is much less sensitive to $T^{*}$ : it increases slightly for $3^{\circ} \mathrm{C} \leq T^{*} \leq 4.5^{\circ} \mathrm{C}$ and decreases, even more slightly, thereafter.

The spatial pattern of the dominant oscillatory modes in both components stays the same for all the values of $T^{*}$ examined here, although their scale and intensity increase when $T^{*}$ increases. The oscillations in the $\mathrm{S}$ component are clearly dominated by barotropic instability (see Fig. 11b and discussion thereof), while those in the A component are dominated by baroclinic instability.

\section{Concluding remarks}

In this paper, we have studied the atmospheric flow induced by an oceanic front, in a minimal baroclinic

TABLE 3. The effect of the oceanic front's strength on the lowfrequency variability in the free atmosphere. The first two columns give the parameter $\alpha$ of Eq. (6) and the frontal gradient $T^{*}$, while the next two columns list the period of the two dominant oscillatory modes in the A and S components. The final column contains the dominant periods of the $\mathrm{S}$ component after low-pass filtering; see text for details.

\begin{tabular}{crccc}
\hline \hline & $\begin{array}{c}T^{*} \\
\left({ }^{\circ} \mathrm{C}\right)\end{array}$ & $\begin{array}{c}\text { A component } \\
\text { (days) }\end{array}$ & $\begin{array}{c}\mathrm{S} \text { component } \\
\text { (days) }\end{array}$ & $\begin{array}{c}\text { S component } \\
\text { filtered } \\
\text { (days) }\end{array}$ \\
\hline 1.032 & 3.0 & 200,85 & 22,44 & 62,45 \\
1.548 & 4.5 & 225,71 & 50,37 & 50,67 \\
2.064 & 6.1 & 270,105 & 30,33 & 71,105 \\
2.580 & 7.6 & 249,145 & 31,28 & 107,71 \\
3.096 & 9.1 & 149,227 & 26,14 & 84,189 \\
3.612 & 10.6 & 77,149 & 14,28 & 100,154 \\
\hline
\end{tabular}

model. The model couples a very simple, linear model of the atmospheric marine boundary layer (AMBL) to a quasigeostrophic (QG), nonlinear model of the free atmosphere with two modes in the vertical; the latter is driven by the vertical velocity at the top of the AMBL. The two-mode model is equivalent to a model with two layers; the connection between two such models is given by Eq. (12), following Flierl (1978) and Feliks and Ghil (1996).

\section{a. Comparison of baroclinic and barotropic results}

The AMBL model here is identical to the one introduced by Feliks et al. (2004, throughout this paper F04) in a model with an even more highly idealized, baro-

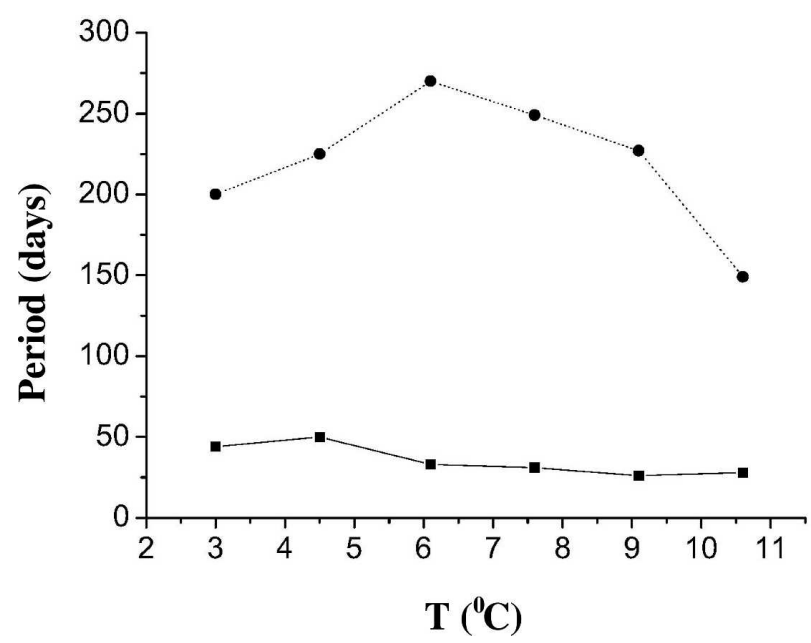

FIG. 14. Period of the dominant oscillatory mode of the symmetric (solid) and antisymmetric components (dotted) as a function of the SST front's strength $T^{*}$, for $\xi=-1$. 
tropic free atmosphere: it has a simple analytical formulation, which permits an explicit solution for the vertical velocity at the top of AMBL. The vertical velocity induced by the oceanic front is proportional to the Laplacian of the sea surface temperature (SST) field. This thermal component approximately balances the mechanical component due to the geostrophic flow above the AMBL; see Eqs. (6) and (7). Based on this simple AMBL formulation, F04 demonstrated the equivalent-barotropic effect of an SST front, while previous work on the atmospheric effect of midlatitude SST anomalies concentrated on shallow baroclinic effects (Doyle and Warner 1990; Kushnir et al. 2002).

The two main questions addressed in the present paper are (i) does the substantial role of the potential vorticity (PV) injection into the free atmosphere survive in a baroclinic model; and (ii) how do baroclinic phenomena modify the mean flow in the free atmosphere induced by the SST front, as well as the lowfrequency variability reported in F04. The answer to the first question is a simple yes: the PV injection effect of the SST front is just as vigorous in the present, the baroclinic model, as in the F04 barotropic one.

Concerning the second question, the atmospheric flow is dominated, here like in F04, by a westerly jet and two antirotating gyres. The mean barotropic mode in both simulations is similar, but the mean jet in the upper layer is stronger (see Figs. 2 and 3) and less stable here, given the same lower-boundary forcing. Already in F04 the symmetry properties of the flow, given the symmetry of the domain and forcing with respect to the horizontal, east-west axis of the domain (see Fig. 1), were important. In F04, the mean atmospheric flow set up by the SST flow had an antisymmetric streamfunction pattern, while the dominant oscillatory instability was, of course, barotropic and had a symmetric pattern. Given the great wealth of instabilities here, we systematically separated the flow into its antisymmetric (A) and symmetric (S) components, to help us identify and describe the various oscillatory modes; see Eq. (16).

We obtained three kinds of unstable oscillatory modes (see Fig. 4). First, A modes that correspond to baroclinic instabilities and have a standing dipole structure (see Fig. 5); the dominant mode of this type has a period of 270 days. Second, S modes develop at the eastern edge of the westerly jet and propagate slowly westward; the dominant mode of this type has a period of 30 days (see Fig. 7). The spatial patterns and periods of these modes are similar to those obtained by F04 and arise by barotropic instabilities of the jet.

Third, we found a northward-propagating wave pattern with a period of 105 days (see Fig. 6). This pattern seems to arise by the interference of two modes of ap- proximately equal period and amplitude: a standing A mode, of baroclinic origin, and an S mode, of barotropic origin, which propagates very slowly westward. This mixed barotropic-baroclinic mode associated with a period of 105 days provides a plausible mechanism for explaining the western North Atlantic 70-day oscillation of Plaut and Vautard (1994).

The period in our model does not agree exactly with the observed one, but the spatiotemporal evolution is fairly realistic. Keppenne et al. (2000) found an oscillatory topographic instability with a 70-day period in a two-layer shallow-water model on the sphere, at a lower horizontal resolution of T21; its spatial pattern, though, was standing, rather than northward propagating.

Our results suggest yet another mechanism for the observed 70-day oscillation, namely the barotropic instabilities that have a period of $60-80$ days and propagate downstream of the jet, as found in F04. Because of the crude spatial resolution, of about $10^{\circ}$, used in Plaut and Vautard (1994), it is difficult at the present stage to draw unambiguous conclusions about which one of the two mechanisms proposed herein is more likely to contribute to the observed oscillation. The Gulf Stream front runs southwest-northeast, rather than west-east, like our idealized oceanic front of Fig. 1. Thus the direction of propagation of the 70-day oscillation observed by Plaut and Vautard (1994) has components both along and perpendicular to the Gulf Stream's north wall. To discriminate between the two mechanisms - the barotropic one found in F04 and the mixed barotropic-baroclinic one found here-one would have to analyze the spatiotemporal features of both the observed and simulated oscillation in greater details. The observational dataset is now longer by a dozen years than that of Plaut and Vautard (1994) and one would need to study it at a finer resolution. Likewise, the modeling results would have to be checked in a configuration forced by a high-resolution SST climatology. We hope to carry out such comparisons in the future.

By tracking the flow's kinetic energy in time (see Fig. 9), two states were identified, while in F04 there was only one such state, for a given value of the thermal forcing $T^{*}$. In the high-energy state, the flow is dominated by two large, counterrotating gyres and a strong westerly jet, which reside in its A component. In the low-energy state, the flow is associated with small gyres and a weak jet. These two energy states thus coincide with the high- and low-energy state of the A component.

The transitions between these two states result from energy fluxes between the A and S components of the 
flow, fluxes that involve baroclinic, as well as barotropic mechanisms (see Figs. 9 and 12). It is the absence of the former that led to the F04 model having a single energy state. The local maximum of the A component coincides with the local minimum of the $\mathrm{S}$ component and vice versa (see Figs. 10 and 11a).

We investigated the dependence of the model's dynamics on the ratio between the depth of the lower layer $\mathrm{H}_{2}$ and the upper layer $H_{1}$ (see Table 2; Fig. 12). The model's baroclinic instabilities are most vigorous when the lower layer is shallow, and are almost entirely absent when the upper layer is shallow. The long-term behavior, though, has its largest baroclinic mode when the two layers are of nearly equal depth.

For small baroclinicity, the symmetric oscillatory modes dominate, while the antisymmetric oscillatory modes are weak. This result is consistent with F04, where only the symmetric modes were present. As the baroclinicity increases, the antisymmetric oscillatory modes become stronger, and the overall mean energy increases, too (see Figs. 10 and 12; Table 2). The period of the dominant oscillatory mode in the A component of the flow reaches its maximum of 270 days for a depth ratio of $H_{2} / H_{1} \simeq 0.4$ (see Fig. 11b; Table 2). In the $\mathrm{S}$ component, the dominant oscillatory mode has a period of about 30 days for all values of $H_{2} / H_{1}$ tested; this value agrees with the ones found in F04, while this mode is stronger here than in F04, for the same forcing.

We also studied systematically (see Table 3; Fig. 14) the dependence of the model's dynamics on the strength $T^{*}$ of the oceanic thermal front or, equivalently, on the parameter $\alpha$ that determines the strength of the vertical velocity at the top of the AMBL; see Eq. (6) and F04. We found that for very weak fronts, $T^{*} \leq$ $1.5^{\circ} \mathrm{C}$, the circulation is steady, like in $\mathrm{F} 04$, and has the same spatial pattern. As the frontal strength increases, $T^{*} \geq 3^{\circ} \mathrm{C}$, the solution becomes chaotic. Thus the westerly jet in the baroclinic, two-mode model is destabilized at lower values of the thermal forcing than in the equivalent-barotropic model and, for the same values of this forcing, the flows are both more intense and more agitated.

\section{$b$. The role of the domain size and horizontal resolution}

To conclude, we supplement this study with two additional numerical experiments, as in F04: in the one we use a larger domain and in the other an increased spatial resolution. First, we carried out an experiment with a domain size of $7000 \mathrm{~km} \times 5000 \mathrm{~km}$, so that the zonal length is larger by $2000 \mathrm{~km}$ than in the previous experiments. We integrated this model version with $T^{*}=$ $6.1^{\circ} \mathrm{C}$ and $\xi=-1$. The mean state of the flow after
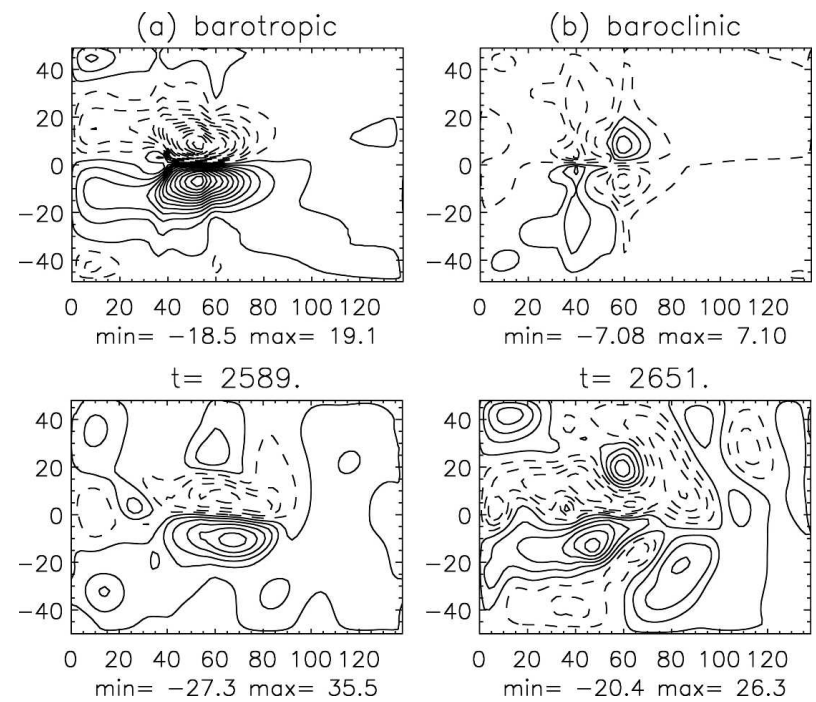

FIG. 15. Model experiment for a $7000 \mathrm{~km} \times 5000 \mathrm{~km}$ basin: (a) barotropic and (b) baroclinic mode of the mean state after spinup; snapshot of the barotropic mode during (c) the high-energy phase, and (d) the low-energy phase.

spinup is shown in Fig. 15a, whereas Figs. 15c,d correspond to snapshots of the barotropic mode during the high-energy and the low-energy state, respectively.

The flow has two large gyres, which occupy the center of the domain and are associated with a strong jet. During the low-energy phase, the flow is characterized by a short jet and two small gyres surrounded by eddies, while the high-energy phase exhibits a longer jet and larger gyres. The spinup and the later evolution for the larger domain size are thus very similar to those in the basic experiment (see Fig. 2).

We considered next the influence of the horizontal grid resolution on the dynamics and carried out several experiments with a spatial resolution of $33 \mathrm{~km} / L$ versus $50 \mathrm{~km} / L$ in the previous experiments. We integrated the model with $\xi=-1$ and examined the solution as a function of the frontal strength $T^{*}=T_{33}$, where the lower index corresponds to the grid interval in kilometers. Following Eq. (23) in F04, the relation between the properly adjusted frontal strengths at the two resolutions is $T_{33} / T_{50}=1.54 / 2.06$.

We ran the model for $T_{33}=3^{\circ}, 4.5^{\circ}$, and $6.1^{\circ} \mathrm{C}$, corresponding to $T_{50}=4.0^{\circ}, 6.1^{\circ}$, and $8.1^{\circ} \mathrm{C}$. The first two prominent periods in the A component are $(150,100)$, $(220,112)$, and $(140,77)$ days, respectively. Comparisons with Table 3 show that the general behavior of the dependency of the period's length on $T^{*}$ is similar in the simulations with the two resolutions: the longest period of the A component has a maximum for $T_{33}=$ $4.5^{\circ} \mathrm{C}\left(T_{50}=6.1^{\circ} \mathrm{C}\right)$, but the periods are somewhat shorter when the spatial resolution is increased. The 
dominant $\mathrm{S}$ component is similar for both grid resolutions and its period is about 30 days.

As in F04, numerical experiments at lower resolutions and without appropriate correction factors for the frontal strength produced only very weak atmospheric responses to an oceanic front of realistic strength. A key conclusion of both studies is, therefore, that high horizontal resolution of the atmospheric model is of the essence in order to capture its response to narrow oceanic fronts, like those associated with eastward jets of the Gulf Stream or Kuroshio Extension type.

Acknowledgments. This work was supported by NSF Grant ATM00-82131 (YF and MG) and DOE Grant DE-FG-020-01ER63251 (MG and ES).

\section{REFERENCES}

Charney, J. G., 1971: Geostrophic turbulence. J. Atmos. Sci., 28, 1087-1095.

— R. Fjortoft, and J. von Neumann, 1950: Numerical integration of the barotropic vorticity equation. Tellus, 2, 237-254.

Doyle, J. D., and T. T. Warner, 1990: Mesoscale coastal processes during GALE IOP 2. Mon. Wea. Rev., 118, 283-308.

Feliks, Y., 1990: Isolated vortex evolution in 2 and 4 mode models. Deep-Sea Res., 37, 571-591.

—, and M. Ghil, 1996: Mixed barotropic-baroclinic eddies growing on an eastward midlatitude jet. Geophys. Astrophys. Fluid Dyn., 82, 137-171.

$\longrightarrow$ - — and E. Simonnet, 2004: Low-frequency variability in the midlatitude atmosphere induced by an oceanic thermal front. J. Atmos. Sci., 61, 961-981.

Flierl, G. R., 1978: Models of vertical structure and the calibration of two-layer models. Dyn. Atmos. Oceans, 2, 341-381.

Fu, L.-L., and G. R. Flierl, 1980: Nonlinear energy and enstrophy transfers in a realistically stratified ocean. Dyn. Atmos. Oceans, 4, 219-246.

Ghil, M., and S. Childress, 1987: Topics in Geophysical Fluid Dynamics: Atmospheric Dynamics, Dynamo Theory and Climate Dynamics. Springer-Verlag, $485 \mathrm{pp}$.

— matic time series. Rev. Geophys., 40, 1003, doi:10.1029/ 2000RG000092.

Haidvogel, D. B., A. R. Robinson, and E. E. Schulman, 1980: The accuracy, efficiency and stability of three numerical models with application to open ocean problems. J. Comput. Phys., 34, 1-53.

Holton, J. R., 1992: An Introduction to Dynamic Meteorology. 3d ed. Academic Press, 511 pp.

Hsu, H., 1987: Study of linear steady atmospheric flow above a finite surface heating. J. Atmos. Sci., 44, 186-199.

Hurrell, J. W., 1995: Decadal trends in the North Atlantic Oscillation: Regional temperatures and precipitation. Science, 269, 676-679.

—_, Y. Kushnir, G. Ottersen, and M. Visbeck, Eds., 2003: The North Atlantic Oscillation: Climatic Significance and Environmental Impact. Geophys. Monogr., Vol. 134, Amer. Geophys. Union.

Jin, F.-F., and M. Ghil, 1990: Intraseasonal oscillations in the ex- tratropics: Hopf bifurcation and topographic instabilities. $J$. Atmos. Sci., 47, 3007-3022.

Keppenne, C. L., and M. Ghil, 1993: Adaptive filtering and prediction of noisy multivariate signals: An application to atmospheric angular momentum. Int. J. Bifurc. Chaos, 3, 625-634.

_ , S. Marcus, M. Kimoto, and M. Ghil, 2000: Intraseasonal variability in a two-layer model and observations. J. Atmos. Sci., 57, 1010-1028.

Kimoto, M., and M. Ghil, 1993a: Multiple flow regimes in the Northern Hemisphere winter. Part I: Methodology and hemispheric regimes. J. Atmos. Sci., 50, 2625-2644.

— , and _ 1993b: Multiple flow regimes in the Northern Hemisphere winter. Part II: Sectorial regimes and preferred transitions. J. Atmos. Sci., 50, 2645-2673.

Kravtsov, S., A. W. Robertson, and M. Ghil, 2005: Bimodal behavior in the zonal mean flow of a baroclinic $\beta$-channel model. J. Atmos. Sci., 62, 1746-1769.

Kushnir, Y., W. A. Robinson, I. Bladé, N. M. J. Hall, S. Peng, and R. Sutton, 2002: Atmospheric GCM response to extratropical SST anomalies: Synthesis and evolution. J. Climate, 15, 22332256.

Lee, T., and P. Cornillon, 1996: Propagation of Gulf Stream meanders between $74^{\circ}$ and $70^{\circ} \mathrm{W}$. J. Phys. Oceanogr., 26, 205224.

Madden, E. A., and P. R. Julian, 1971: Detection of a 40-50 day oscillation in the zonal wind in the tropical Pacific. J. Atmos. Sci., 28, 702-708.

Mann, M. E., and J. M. Lees, 1996: Robust estimation of background noise and signal detection in climatic time series. Climatic Change, 33, 409-445.

Moron, V., R. Vautard, and M. Ghil, 1998: Trends, interdecada and interannual oscillations in global sea-surface temperatures. Climate Dyn., 14, 545-569.

Pedlosky, J., 1987: Geophysical Fluid Dynamics. 2d ed. SpringerVerlag, $710 \mathrm{pp}$.

Plaut, G., and R. Vautard, 1994: Spells of low-frequency oscillations and weather regimes in the Northern Hemisphere. $J$. Atmos. Sci., 51, 210-236.

Rhines, P. B., 1975: Waves and turbulence on a beta-plane. $J$. Fluid Mech., 69, 417-443.

Robertson, A. W., M. Ghil, and M. Latif, 2000: Interdecadal changes in atmospheric low-frequency variability with and without boundary forcing. J. Atmos. Sci., 57, 1132-1140.

Salmon, R., 1998: Lectures on Geophysical Fluid Dynamics. Oxford University Press, $378 \mathrm{pp}$.

Simmons, A. J., J. M. Wallace, and G. W. Branstator, 1983: Barotropic wave propagation and instability, and atmospheric teleconnection patterns. J. Atmos. Sci., 40, 1363-1392.

Stommel, H. M., 1965: The Gulf Stream: A Physical and Dynamical Description. 2d ed. University of California Press, 248 pp.

- and K. Yoshida, Eds., 1972: Kuroshio: Physical Aspects of the Japan Current. University of Washington Press, 517 pp.

Sweet, W., R. Fett, J. Kerling, and P. LaViolette, 1981: Air-sea interaction effects in the lower troposphere across the north wall of the Gulf Stream. Mon. Wea. Rev., 109, 1042-1052.

Thomson, D. J., 1982: Spectrum estimation and harmonic analysis. Proc. IEEE, 70, 1055-1096.

Warner, T. T., M. N. Lakhtakia, J. D. Doyle, and R. A. Pearson, 1990: Marine atmospheric boundary layer circulations forced by Gulf Stream sea surface temperature gradients. Mon. Wea. Rev., 118, 309-323. 